\title{
Several Routes to Determining Entropy Generation in The Early Universe
}

\author{
A. Beckwith \\ Chongqing University Department of Physics: Chongqing University, Chongqing, PRC, 400044 \\ *Corresponding Author: abeckwith@uh.edu
}

Copyright $@ 2013$ Horizon Research Publishing All rights reserved.

\begin{abstract}
We analyze how entropy could be generated via a semi classical argument as well as by multiple brane- anti brane combinations leading to an initial soliton-instanton formation. The supposition is that the two different types of methods give similar initial conditions for entropy and information/ computational bits of information in the initial universe. We close then with observations we think are pertinent to entropy increase and also the variation of statistical noise about the CMBR spectra. This is linkable to a table of computational bits as presented by Smoot in 2007. We also have a tie in with the race track model of inflation in the cooling down period, as other fields to which the inflaton couples through gravity undergo symmetry breaking phase transitions as the universe cools. This article will set the stage for later evaluating how brane-anti brane models of entropy fare against more classical formulations of entropy in a future publication.
\end{abstract}

Keywords Inflaton, brane-anti brane, entropy, statistical noise, CMBR spectra

PACS 96.50.Ry, 98.80.-k, 98.80.Cq, 98.80.Bp

\section{Introduction}

We wish to present two alternative routes to generation of entropy. One is strictly a semi classical argument, linked to the exponential of early universe entropy being proportional to the spatial integral of energy minus an interaction between particles or clumps 1 . The other is a brane world summation of brane and anti brane components 2 which are in a very small space time geometry of no less than several orders of magnitude larger than Planck length, cubed. This second result is orthodox brane theory for the formation of an instanton, but it is remarkable that this same argument as given in the IUCAA meeting pre supposes the same increase in minimum geometrical length scale which the more semi classical argument gives.

We then will mention how to form a five dimensional instanton in pre inflation space3, and then how to form worm hole bridge between a prior to our present universe. This will be put in the context of an embedding of the instanton in pre inflation space, and its connections with the model given above of brane world instanton formation due to branes and anti branes. This specific formulation of the wormhole bridge combined with instantons has consequences to black hole physics and entropy via brane and anti branes 4 which we will mention as an area to be explored later.

The intersection of all these arguments achieves several goals simultaneously. First, it establishes a minimum criteria for forming entropy. It secondly links entropy to instanton formation. We also mention an argument linked to changes in energy density states, at the start of inflation with an increase as of entropy which may account for the dramatic growth of entropy after a causal discontinuity barrier breaking the instanton of energy density transmitted from a prior to a present universe.

The main point of the article which is presented toward the end is that there are bench marks as to information based complexity of cosmological evolution. This was presented by Smoot in the Challonge symposia of August 20075. The main point in the end is that analyzing the particulars of cosmological models which purport to analyze initial phases of the big bang, and inputs of the big bang would be well advised to answer or in part match the computational complexity bench marks

1 Padmanabhan, T, “ Theoretical Astro physics, Volume 1 : Astrophysical processes “, Cambridge University Press, Cambridge, UK, 2000

2 Mathur, S., and Chowdhury, B., "Fractional Brane States in the Early Universe”, Classical and Quantum Gravity, 24 (2007), pp. 2689-2720

3 Wesson, P., " Space-Time-Matter, Modern Kaluza Klein Theory”, World Scientific , Singapore, Republic of Singapore, 1999

4 Lifschytz, G. "Black Hole thermalization rate from brane anti brane model ", arXIV hep-th/ /0406203 v1 23 june, 2004

5 Smoot, G, 11 th Paris Cosmology Colloquium, August 18th, 2007 with respect to Smoot, G, "CMB Observations and the Standard Model of the Universe"

'D.Chalonge' school, http://chalonge.obspm.fr/Programme2007.html 
Smoot is offering in the table reproduced at the end of this document.

In addition, a direct comparison of the relative importance of the different entropy formulations will be written up as a sequel to these results. I.e. a comparison of the string theory results as compared to more classical formulations comes out later.

\section{Semi Classical Models of Entropy Generation}

Kolb and Turner 6 have a temperature $T$ related entropy density which can be treated as being written as :

$$
S_{\text {Density }}=\frac{2 \cdot \pi^{2}}{45} \cdot g_{*} \cdot T^{3}
$$

This pre supposes when we do it that we are able to state a total entropy as the entropy density times space time volume $V_{4}$

$$
S_{\text {total }} \equiv S_{\text {Density }} \cdot V_{4}
$$

In this situation we are writing for initial conditions with a temperature $T \approx 10^{32} \mathrm{~K}$ for the initiation of quantum effects for quantum gravity as given by Weinberg 7 .

$T \approx 10^{32} \mathrm{~K} \approx 1.3 \times 10^{28} \mathrm{eV} \sim 1.3 \times 10^{19} \mathrm{GeV}$. This gives us the option of comparing what we get in entropy with Seth Lloyds8

$$
I=S_{\text {total }} / k_{B} \ln 2=[\# \text { operations }]^{3 / 4}=\left[\rho \cdot c^{5} \cdot t^{4} / \hbar\right]^{3 / 4}
$$

We will examine if or not the following is actually true in terms of time, i.e. can we write $I=\left(t / t_{P}\right)^{2}$ ? This is assuming that the density9 $\rho \equiv T^{00} \sim \Lambda_{\text {vacumm-energy }}$ which is initially enormous, and which will be due in terms of a transfer of energy density from a prior universe to our present universe, which will be elaborated upon later in this document.

We can if we take the absolute value of Eq. (3) and (2) above get for small volume values good estimates as to the relative volume of the phase space in early universe cosmology where Eq. (2) and Eq. (3) are congruent with each other. For our purposes, we will take time as greater than (or equal) to a Planck time interval, in line with the temperature dependence of entropy density mentioned in Eq. (1) above.

We can compare this with Padamanadan's treatment of entropy ${ }^{1}$ which is with regards to micro canonical ensemble as defined via

$$
\begin{aligned}
& \exp \left(S_{\text {total }}\right)=g(E)=\frac{A}{N !} \cdot \int d^{3 N} x \cdot\left[E-\frac{1}{2} \cdot \sum_{i \neq j} U\left(x_{i}, x_{j}\right)\right]^{\frac{3 N}{2}} \\
& \leq \frac{A}{N !} \cdot \int d^{3 N} x \cdot[E]^{\frac{3 N}{2}} \approx\left[\frac{A}{N !} \cdot \int d^{3 N} x\right] \cdot\left[\frac{\Lambda_{M a x} V_{4}}{8 \cdot \pi \cdot G}\right]^{\frac{3 N}{2}}
\end{aligned}
$$

If $A \sim O(1)$, i.e. we re scale it as being of order unity, and $N \sim 10^{87}$ particles, and we re scale $\int d^{3 N} x \sim V_{4}^{N}$ where we choose $V_{4}$, and where we assume Eq. (2) and Eq. (3) are equivalent and we assume that there is grounds for writing $\frac{\Lambda_{M a x} V_{4}}{8 \cdot \pi \cdot G} \sim T^{00} V_{4} \equiv \rho \cdot V_{4} \gg \frac{1}{2} \cdot \sum_{i \neq j} U\left(x_{i}, x_{j}\right)$, we can shed light on if or not it is still feasible to treat entropy, with $N \sim 10^{87}$ as a micro canonical ensemble phenomena, which we claim has implications for the formation of an instanton in early universe cosmology. Frankly we would want, in early universe cosmology that we have $\rho \cdot V_{4} \neq \frac{1}{2} \cdot \sum_{i \neq j} U\left(x_{i}, x_{j}\right)$, but not by too much, so we can form an instanton.

6 Kolb, E., and Turner, S. “ The Early Universe”, Westview Press, Chicago, USA, 1994

7 Weinberg, S., Gravitation and Cosmology: Principles and Applications of the General theory of Relativity, John Wiley \& Sons, Inc. New York, 1972

8 Lloyd, S., "Computational capacity of the universe", Phys. Rev. Lett. 88, 237901 (2002)

9 Beckwith, A. "Symmetries in evolving spacetime from prior to present unverses", arXIV math-ph/0501028 (2007) 


\section{Brane World Picture of Early Universe Entropy Formation}

This discussion is adapted from a lecture given at the ICGC-07 conference by Samir Mathur ${ }^{2}$. The supposition is that branes and anti branes form the working component of an instanton.

I.e. look at the case, first of massless radiation, and then we obtain for D space time dimensions, and E the general energy

$$
S \sim E^{(D-1 / D)}
$$

This has a further development as:

$$
\frac{\Lambda_{M a x} V_{4}}{8 \cdot \pi \cdot G} \sim T^{00} V_{4} \equiv \rho \cdot V_{4}=E_{\text {total }}
$$

The question now becomes how do we go about defining what the necessary volume is re scaled via a quantum gravity changing of how to measure gravitational lengths which are for the threshold of quantum gravity. Traditionally the bench marking has been via the Planck length $l_{P} \sim 10^{-33} \mathrm{~cm} \underset{\text { Quantum-Gravity-threshold }}{\longrightarrow} \widetilde{N}^{\alpha} \cdot l_{P}$. This re scaling of the minimum length needed for the importance of quantum gravity effects showing up in a grid of space time resolves, as information paradox of black hole physics. So far we have merely been working with a typical string gas model for entropy. Now, let us add in a supposition for $\breve{N}$ branes and anti branes to put in an instanton structure as to how we look at the entropy. Lifschytz ${ }^{4}$ in 2004 codified thermalization equations of the black hole which was recovered from the model of branes and anti-branes, and in lieu of assuming an anti brane is merely the charge conjugate of say a Dp brane wrote an entropy along the lines of modifying Eqn. (5) to read

$$
S_{\text {Total }} \sim \breve{a} \cdot\left[\frac{E_{\text {Total }}}{2^{n}}\right]^{\lambda} \cdot \prod_{j=1}^{N}\left(\sqrt{M_{p j, 0}}+\sqrt{M_{\bar{p} j, 0}}\right)
$$

This has when we do it $E_{\text {Total }}$ as in Eqn. (5) above, and proportional to the cosmological vacuum energy parameter. Of course, in string theory, the energy is also defined via

$$
E_{\text {Total }}=4 \lambda \cdot \sqrt{M_{p j, 0} \cdot M_{\bar{p} j, 0}}
$$

Furthermore, the values of $M_{p j, 0}$, and $M_{\bar{p} j, 0}$ refer to the mass of $\mathrm{p}$ branes and $\mathrm{p}$ anti branes,_as Gilad Lifschytz refers to it.

This treatment can be changed and rescaled to treating the mass and the energy of the brane contribution along the lines of Mathur's ${ }^{2}$ CQG article where he has a string winding intepretation of energy along the lines of putting as much energy $E$ into string windings as possible via, $\left[n_{1}+\bar{n}_{1}\right] L T=\left[2 n_{1}\right] L T=E / 2$, where we are talking about $n_{1}$ wrappings of a string about a cycle of the torus, and $\bar{n}_{1}$ being 'wrappings the other way', with the torus having a cycle of length L, which leads to an entropy defined in terms of an energy value of, if mass $m_{i}=T_{P} \prod L_{j}$ (with $T_{P}$ being the tension of the $i$ th brane, and $L_{j}$ being spatial dimensions of a complex torus structure )

$$
E_{\text {Total }}=2 \sum_{i} m_{i} n_{i}
$$

This leads to entropy

$$
S_{\text {Total }}=A \cdot \prod_{i}^{N} \sqrt{n_{i}}
$$

Our claim is that this very specific value of entropy for Eqn. (9) above will in Planck interval of time at about the onset of inflation lead to

$$
\left|\left[S_{\text {Total }}=A \cdot \prod_{i}^{N} \sqrt{n_{i}}\right] / k_{B} \ln 2\right| \approx[\text { \# operations }]^{3 / 4} \approx 10^{8}
$$

Furthermore we also claim that the interaction of the branes and anti branes will form an instanton structure, which is implicit in the treatment outlined in Eq. (7), and that the numerical counting given in Eq. (9) merely reflects that branes and anti branes, even if charge conjugates of each other have the same 'wrapping number' $n_{i}$. 


\section{Specific numerical estimates for the minimum boundary of quantum gravity volume vs. classical gravity dominated effects}

We begin with a temperature estimate of $T \approx 10^{45} \mathrm{~K}>T_{\text {QG-Threshold }} \sim 10^{32} \mathrm{~K}$. Then, Eq. (4) above modified when we take the absolute value will lead to, if we look at when $N \approx 10^{86}$ :

$$
\left|S_{\text {Total }}\right| \sim\left|k_{B} \cdot \ln 2\right| \cdot[\# \text { operations }]^{3 / 4} \approx N\left|-\log \frac{N}{10}+\log V_{4}^{3}+\log E^{3 / 2}\right| \sim 10^{8}
$$

Leading to solving for $\mathrm{E}$ as follows $\Rightarrow \rho \cdot V_{4} \neq \frac{1}{2} \cdot \sum_{i \neq j} U\left(x_{i}, x_{j}\right)$, and also that

$$
V_{4}^{3} \cdot E^{3 / 2} \sim 10^{85} \Rightarrow E \sim \frac{10^{57}}{V_{4}^{2}} \equiv \frac{\Lambda_{M a x} \cdot V_{4}}{8 \cdot \pi \cdot G}
$$

We can and will reference what we can say about $\Lambda_{\operatorname{Max}} \sim c_{2} \cdot T^{\tilde{\beta}}$, as given by Park (2003), as a way to get an upper bound estimate upon $V_{4}$ for quantum gravity effects in inflation. We get an upper bond estimate of

$$
\left.V_{4}\right|_{\text {Threshold-volume-for-quantum-effects }} \sim 10^{-4} \mathrm{~cm}
$$

This is way too large, but it indicates that the interaction of material within the region of space being considered does not obey $\rho \cdot V_{4} \gg \frac{1}{2} \cdot \sum_{i \neq j} U\left(x_{i}, x_{j}\right)$. If this is what we have, we can then begin to look at if the instanton picture is true or not. We will first review what can be said about different variants of vacuum energy. I.e. where the vacuum energy models of four and five dimensions could conceivably overlap. But to do this we will look at what these models are.

\section{Comparing different models for how one can input thermal-radiation energy}

Begin first with looking at different value of the cosmological vacuum energy parameters, in four and five dimensions ${ }^{9}$.

$$
\left|\Lambda_{5-\operatorname{dim}}\right| \approx c_{1} \cdot\left(1 / T^{\alpha}\right)
$$

in contrast with the more traditional four dimensional version of the same, minus the minus sign of the brane world theory version The five dimensional version is actually connected with Brane theory, and higher dimensions, whereas the four dimensional is linked to more traditional De Sitter space time geometry, as given by Park10 (2003)

$$
\Lambda_{4-\operatorname{dim}} \approx c_{2} \cdot T^{\beta}
$$

This is such that If one looks at the range of allowed upper bounds of the cosmological constant, we have that the difference between what Barvinsky11 (2006) recently predicted, and Park (2003)

$$
\Lambda_{4-\operatorname{dim}} \propto c_{2} \cdot T \underset{\text { graviton-production }}{\longrightarrow} 360 \cdot m_{P}^{2}<<c_{2} \cdot\left[T \approx 10^{32} \mathrm{~K}\right]
$$

Needless to say, right after the gravitons are released one still is seeing a drop off of temperature contributions to the cosmological constant. Then we can write, for small time values $t \approx \delta^{1} \cdot t_{P}, 0<\delta^{1} \leq 1$ and for temperatures sharply lower than $T \approx 10^{12}$ Kelvin, Beckwith ${ }^{9}$ (2007), where for a positive integer $n$

$$
\frac{\Lambda_{4-\operatorname{dim}}}{\left|\Lambda_{5-\operatorname{dim}}\right|}-1 \approx \frac{1}{n}
$$

If we have an order of magnitude equivalence between such representations, we can talk about a quantum regime of gravity which is consistent with regards to fluctuations in energy and also in the growth of entropy. We will use an order of magnitude estimate as to presenting what the vacuum energy should be in the neighborhood of Planck time in the advent of

10 Park, D.K., Kim, H., and Tamarayan, S., "Nonvanishing Cosmological Constant of Flat Universe in Brane world Senarios,” Phys.Lett. B535 (2002) pp. $5-10$

11 Barvinsky,A., Kamenschick,A., Yu, A. , “ Thermodynamics from Nothing: Limiting the Cosmological Constant Landscape, Phy Rev D 74121502 (Rapid communications) 
nucleation of a new universe.

\section{First principles argument as to large scale values of the absolute magnitude of the cosmological vacuum energy}

Look at an argument provided by Padmanabhan12, leading to the observed cosmological constant value suggested by Park. Assume that $l_{P} \sim 10^{-33} \mathrm{~cm} \underset{\text { Quantum-Gravity-threshold }}{\longrightarrow} \widetilde{N}^{\alpha} \cdot l_{P}$, but that when we make this substitution that $1 \leq \widetilde{N}^{\alpha} \leq 10^{2} 13$

$$
\begin{gathered}
\rho_{V A C} \sim \frac{\Lambda_{\text {observed }}}{8 \pi G} \sim \sqrt{\rho_{U V} \cdot \rho_{I R}} \\
\sim \sqrt{l_{\text {Planck }}^{-4} \cdot l_{H}^{-4}} \sim l_{\text {Planck }}^{-2} \cdot H_{\text {observed }}^{2} \\
\Delta \rho \approx \text { a dark energy density } \sim H_{\text {observed }}^{2} / G
\end{gathered}
$$

We can replace $\Lambda_{\text {observed }}, H_{\text {observed }}^{2}$ by $\Lambda_{\text {initial }}, H_{\text {initial }}^{2}$. In addition we may look at inputs from the initial value of the Hubble parameter to get the necessary e folding needed for inflation, according to

$$
\begin{aligned}
& E-\text { foldings }=H_{\text {initial }} \cdot\left(t_{\text {End of inf }}-t_{\text {beginning of inf }}\right) \equiv N \geq 100 \\
& \Rightarrow H_{\text {initial }} \geq 10^{39}-10^{43}
\end{aligned}
$$

Leading to

$$
a(\text { End }- \text { of }-\mathrm{inf}) / a(\text { Beginning }- \text { of }-\inf ) \equiv \exp (N)
$$

If we set $\Lambda_{\text {initial }} \sim c_{1} \cdot\left[T \sim 10^{32}\right.$ Kelvin $\rfloor$ implying a very large initial cosmological constant value, we get in line with what Park suggested for times much less than the Planck interval of time at the instant of nucleation of a vacuum state

$$
\Lambda_{\text {initial }} \sim\left[10^{156}\right] \cdot 8 \pi G \approx \text { huge number }
$$

Question. Do we always have this value of Eq. (22)? At the onset of Inflation? When we are not that far away from a volume of space characterized by $l_{P}^{3}$, or at most 100 or so times larger ? Contemporary big bang theories imply this. I.e. a very high level of thermal energy. We need to ask if this is something which could be transferred from a prior universe, i.e. could there be a pop up nucleation effect, i.e. emergent space time? Appendix 1 gives a way for this to occur. We will now examine a mechanism which would allow for this to happen. It involves transfer of energy from a prior to the present universe.

\section{Worm hole transition from a prior to the present universe}

To model this, we use results from Crowell 14 on quantum fluctuations in space time which gives a model from a pseudo time component version of the Wheeler De Witt equation, with use of the Reinssner-Nordstrom metric to help us obtain a solution which passes through a thin shell separating two space times. The radius of the shell, $r_{0}(t)$ separating the two space times is of length $l_{P}$ in approximate magnitude, leading to a domination of the time component for the Reissner - Nordstrom metric

$$
d S^{2}=-F(r) \cdot d t^{2}+\frac{d r^{2}}{F(r)}+d \Omega^{2}
$$

This has:

12 Padmanabhan, T., “An Invitation to Astro physics”, World Scientific Publishing Co. Pte. Ltd, Singapore, 2006

13 Padmanabhan, T." Understanding Our Universe: Current Status and Open Issues”, in 100 Years of Relativity Space-Time Structure: Einstein and Beyond, edited by Ashtekar, A., World Press Scientific, Singapore, 2005

14 Crowell, L, “Quantum Fluctuations of Space Time “, World Scientific Series in Contemporary Chemical Physics, Vol 25, World Scientific, PTE, LTD, 2005, Singapore 


$$
F(r)=1-\frac{2 M}{r}+\frac{Q^{2}}{r^{2}}-\frac{\Lambda}{3} \cdot r^{2} \underset{T \rightarrow 10^{32} \text { Kelvin } \sim \infty}{\longrightarrow}-\frac{\Lambda}{3} \cdot\left(r=l_{P}\right)^{2}
$$

This assume that the cosmological vacuum energy parameter has a temperature dependence as outlined by Park (2003) leading to if

$$
\frac{\partial F}{\partial r} \sim-2 \cdot \frac{\Lambda}{3} \cdot\left(r \approx l_{P}\right) \equiv \eta(T) \cdot\left(r \approx l_{P}\right)
$$

As a wave functional solution to a Wheeler De Witt equation bridging two space times. This solution bridging two space times is similar to that being made between these two space times with 'instantaneous' transfer of thermal heat ,as given by Crowel1 ${ }^{14}(2005)$

$$
\Psi(T) \propto-A \cdot\left\{\eta^{2} \cdot C_{1}\right\}+A \cdot \eta \cdot \omega^{2} \cdot C_{2}
$$

This has $C_{1}=C_{1}(\omega, t, r)$ as a pseudo cyclic and evolving function in terms of frequency, time, and spatial function, with the same thing describable about $C_{2}=C_{2}(\omega, t, r)$ See Appendix I below as to what the entries in Eqn. (26) mean above.

\section{Interesting speculation. Does there exist a five dimensional version of an instanton in the worm hole transition regime?}

We will attempt to build the contribution as to a Reissner-Nordstrom metric embedded in a five dimensional space- time metric, and see if this satisfied . Eq. (27) This allows us to determine, using of the Risessner-Nordstrom metric as given, by Kip Thorne, Wheeler, and Misner 15, for an added cosmological 'constant' $\Lambda$ and 'charge' $Q$

This will be shown to lead to 16

$$
\begin{gathered}
M_{g}(r)=\int\left[T_{0}^{0}-\left(T_{1}^{2}+2 \cdot T_{2}^{2}\right)\right] \cdot \sqrt{-g_{4}} d V_{3} \\
\approx \pi \cdot c_{1}^{2} \cdot\left[\frac{r^{3}}{3}-2 M \cdot \frac{r^{2}}{2}+Q \cdot r-\frac{\Lambda}{15} \cdot r^{5}\right]+ \\
4 \pi \cdot c_{1} \cdot\left[r^{2}-8 \cdot M \cdot r-\frac{\Lambda}{3} \cdot r^{4}\right] \underset{r \rightarrow \delta}{\longrightarrow} \varepsilon^{+} \approx 0
\end{gathered}
$$

To do this, we start off with the following space time line metric in five dimensions. This is a modification of Wesson's book $^{16}$.

$$
\begin{aligned}
& d S_{5-\operatorname{dim}}=[\exp (i \pi / 2)] \cdot\left\{\begin{array}{l}
e^{2 \Phi(r)} d t^{2} \\
+e^{2 \widetilde{\Lambda}(r)} d r^{2}+R^{2} d \Omega^{2}
\end{array}\right\} \\
& +(-1) \cdot e^{\mu} d l^{2}
\end{aligned}
$$

We claim that what is in the \{\} brackets is just the Reissner-Nordstrom line metric in four dimensional space. The parameters in the \{\} bracket are linked to the Reissner-Nordstrom metric via

$$
e^{2 \Phi(r)}=\left(1-\frac{2 M}{r}+\frac{Q^{2}}{r^{2}}\right)
$$

And

$$
e^{2 \widetilde{\Lambda}(r)}=\left(1-\frac{2 M}{r}+\frac{Q^{2}}{r^{2}}\right)^{-1}
$$

And this is assuming that $\quad R \sim r$ as well as using $\mu \approx c_{1} \cdot r$ with a maximum value topped off by a Planck's length value 
due to $\mu_{\text {Maximum }} \approx c_{1} \cdot r_{\text {Maximum }} \sim l_{P} \equiv 10^{-35} \mathrm{~cm}$. So being the case, we get the following stress tensor values

$$
\begin{array}{r}
T_{0}^{0}=\left(\frac{-1}{8 \pi}\right) \cdot\left(1-\frac{2 M}{r}+\frac{Q^{2}}{r^{2}}-\frac{\Lambda}{3} r^{2}\right) \cdot\left(\frac{c_{1}^{2}}{4}+\frac{c_{1}}{r}+\frac{c_{1}}{4} \cdot\left[\frac{\frac{2 M}{r^{2}-\frac{2 Q}{r^{3}}-\frac{2 \Lambda r^{2}}{3}}}{1-\frac{2 M}{r}+\frac{Q^{2}}{r^{2}}-\frac{\Lambda}{3} r^{2}}\right]\right) \\
T_{1}^{1}=\left(\frac{-1}{8 \pi}\right) \cdot\left(1-\frac{2 M}{r}+\frac{Q^{2}}{r^{2}}-\frac{\Lambda}{3} r^{2}\right) \cdot\left(\frac{c_{1}}{r}+\frac{c_{1}}{4} \cdot\left[\frac{\frac{2 M}{r^{2}}-\frac{2 Q}{r^{3}}-\frac{2 \Lambda r^{2}}{3}}{1-\frac{2 M}{r}+\frac{Q^{2}}{r^{2}}-\frac{\Lambda}{3} r^{2}}\right]\right) \\
T_{2}^{2}=T_{3}^{3}=\left(\frac{-1}{8 \pi}\right) \cdot\left(1-\frac{2 M}{r}+\frac{Q^{2}}{r^{2}}-\frac{\Lambda}{3} r^{2}\right) \cdot\left(\frac{c_{1}^{2}}{4}+\frac{c_{1}}{r}+\frac{c_{1}}{2} \cdot\left[\frac{\frac{2 M}{r^{2}}-\frac{2 Q}{r^{3}}-\frac{2 \Lambda r^{2}}{3}}{1-\frac{2 M}{r}+\frac{Q^{2}}{r^{2}}-\frac{\Lambda}{3} r^{2}}\right]\right)
\end{array}
$$

Furthermore, we get the following determinant value

$$
\sqrt{-g_{4}}=\left(1-\frac{2 M}{r}+\frac{Q^{2}}{r^{2}}-\frac{\Lambda}{3} r^{2}\right)
$$

All these together lead to Eq. (27) being satisfied. Let us now see how this same geometry contributes to a worm hole bridge and a solution as to forming the instanton flux wave functional between a prior to a present universe. The Reissner-Nordstrom metric permits us to have a radiation dominated 'matter' solution whose matter 'contribution' drops off rapidly as the spatial component of geometry goes to zero. This is in tandem with radiation pressure and density falling off rapidly, as we leave the center of such a purported soliton/ instanton. This is extremely useful because it ties in with the notion of fractional branes contributing to entropy calculations. In fact it is useful to state that these two notions dove tail with each other quite closely. The only difference is that the construction above does not in itself lend to the complexity of what we would observe, which is in itself a multiple - joined net work of charge centers and of shifting geometry.

\section{Energy fluctuations due to the worm hole and their link to entropy fluctuations}

We argue that the existence of the worm hole and an instanton formation in the throat of the worm hole will lead to a constant energy flux. Note that we are assuming a constant energy flux through the worm hole. This is equivalent to work with an expression given by Mukhanov17 about energy density fluctuations and entropy. In position space, it is for energy density $\rho(x)$, and entropy $S(x)$

$$
\frac{\partial^{2} \delta \rho(x)}{\partial t^{2}}-c_{s}^{2} \Delta \cdot \delta \rho(x)-4 \pi \cdot G \rho_{0} \cdot \delta \rho(x)=\sigma \cdot \Delta \delta S(x)
$$

This is Fourier transformed into being

$$
\frac{\partial^{2} \delta \rho(k)}{\partial t^{2}}+k^{2} c_{s}^{2} \cdot \delta \rho(k)-4 \pi \cdot G \rho_{0} \cdot \delta \rho(k)=-\sigma \cdot k^{2} \delta S(k)
$$

This has a time independent solution of the form given by, assuming small spatial dimensions

$$
\delta \rho(k) \equiv-\frac{\sigma k^{2} \delta S(k)}{\left(k^{2} c_{s}^{2}-4 \pi G \rho_{0}\right)} \underset{k \rightarrow b i g}{\longrightarrow}-\frac{\sigma \delta S(k)}{\left(c_{s}^{2}\right)}
$$


This may be Fourier transformed, assuming near constant values of $\mathrm{k}$ and position $\mathrm{x}$, to be in $\mathrm{x}$ position space

$$
\delta \rho(x) \cong-\frac{8 \sigma}{c_{s}^{2}} \delta S(x)
$$

Here, $c_{s}^{2}$ is the square of the speed of sound which is in early universe conditions close to unity. We also have that $\sigma \equiv(\partial p / \partial S)_{\rho}$. Then we can state that when we have $\delta \rho(x) \propto \Lambda_{\text {initial }} \rightarrow \Lambda_{\max }$ due to increasing temperature

$$
|\delta \rho(x)| \cong 8 \sigma \cdot \delta S(x)
$$

We claim that the increase in entropy, is connected with a breaking of the instanton structure of a packet of energy transferred from a prior space time to our own. Let us see how this could come about.

\section{What leads to caual discontinuity in scale factor evolution}

The Friedmann equation 18 for the evolution of a scale factor $a(t)$,

$$
(\dot{a} / a)^{2}=\frac{8 \pi G}{3} \cdot\left[\rho_{r e l}+\rho_{\text {matter }}\right]+\frac{\Lambda}{3}
$$

suggests a non partially ordered set evolution of the scale factor with evolving time, thereby implying a causal discontinuity. The validity of this formalism is established by rewriting the Friedman equation as follows $19^{\prime}: a\left(t^{*}\right)<l_{P}$ for $t^{*}<t_{P}=$ Planck time, and $a_{0} \equiv l_{P}$, for a discrete equation model of Eq. (40) leads to

$$
\begin{aligned}
& {\left[\frac{a\left(t^{*}+\delta t\right)}{a\left(t^{*}\right)}\right]-1<} \\
& \frac{\left(\delta t \cdot l_{P}\right)}{(\sqrt{3 / 8 \pi \Lambda})} \cdot\left[\frac{1}{24 \pi \cdot a^{2}\left(t^{*}\right)}+\frac{1}{\Lambda} \cdot\left[\left(\rho_{r e l}\right)_{0} \cdot \frac{a_{0}^{4}}{a^{6}\left(t^{*}\right)}+\left(\rho_{m}\right)_{0} \cdot \frac{a_{0}^{3}}{a^{5}\left(t^{*}\right)}\right]\right]^{1 / 2} \\
& \underset{\delta t \rightarrow \varepsilon^{+}, \Lambda \neq \infty, a \neq 0}{\longrightarrow}\left(\frac{\delta t \cdot\left[l_{P} / a\left(t^{*}\right)\right]}{\sqrt{3 / 8 \pi}}\right) \cdot \sqrt{\frac{\left(\rho_{r e l}\right)_{0} a_{0}^{4}}{a^{4}\left(t^{*}\right)}+\frac{\left(\rho_{m}\right)_{0} a_{0}^{3}}{a^{3}\left(t^{*}\right)}} \approx \varepsilon^{+}<<1
\end{aligned}
$$

So in the initial phases of the big bang, with very large vacuum energy $\neq \infty$ and $a\left(t^{*}\right) \neq 0,0<a\left(t^{*}\right)<<1$, the following relation, which violates (signal) causality, is obtained for very small fluctuation $a\left(t^{*}\right)<l_{P}$ for $t^{*}<t_{P}=$ Planck time, and $a_{0} \neq l_{P}, a_{0}>l_{P}$, which indicates that ${ }^{18}$

$$
\rho_{\text {rel }} \equiv\left(\frac{a_{\text {present-era }}}{a(t)}\right)^{4} \cdot\left(\rho_{\text {rel }}\right)_{\text {present-era }}
$$

And

$$
\rho_{m} \equiv\left(\frac{a_{\text {present-era }}}{a(t)}\right)^{3} \cdot\left(\rho_{m}\right)_{\text {present-era }}
$$

Using this above creates the following as plausible estimates which can be reviewed as needed. For large but not infinite temperatures, and for $\Lambda \sim c_{1} T^{\alpha}$

18 Frampton, P, Baum, L, “Turnaround in Cyclic Cosmology “,Phys.Rev.Lett. 98 (2007) 071301 , arXiv:hep-th/0610213v2 


$$
\left(\frac{\delta t \cdot\left[l_{P} / a\left(t^{*}\right)\right]}{\sqrt{3 / 8 \pi}}\right) \cdot \sqrt{\frac{\left(\rho_{r e l}\right)_{0} a_{0}^{4}}{a^{4}\left(t^{*}\right)}+\frac{\left(\rho_{m}\right)_{0} a_{0}^{3}}{a^{3}\left(t^{*}\right)}} \sim 10^{-45} \cdot 10^{1} \cdot \sqrt{10^{80}} \approx 10^{-4}<<1
$$

If we examine what happens with $\left|\Lambda_{5-\text { dim }}\right| \sim c_{2} T^{-\beta}$

$$
\begin{aligned}
& \frac{\left(\delta t \cdot\left[l_{P} / a\left(t^{*}\right)\right]\right)}{(\sqrt{3 / 8 \pi \Lambda})} \cdot\left[\frac{1}{24 \pi}+\frac{1}{\Lambda} \cdot\left[\left(\rho_{r e l}\right)_{0} \cdot \frac{a_{0}^{4}}{a^{4}\left(t^{*}\right)}+\left(\rho_{m}\right)_{0} \cdot \frac{a_{0}^{3}}{a^{3}\left(t^{*}\right)}\right]\right]^{1 / 2} \\
& \sim\left(\frac{\delta t \cdot\left[l_{P} / a\left(t^{*}\right)\right]}{\sqrt{3 / 8 \pi}}\right) \cdot \sqrt{\frac{\left(\rho_{r e l}\right)_{0} a_{0}^{4}}{a^{4}\left(t^{*}\right)}+\frac{\left(\rho_{m}\right)_{0} a_{0}^{3}}{a^{3}\left(t^{*}\right)}} \sim 10^{-45} \cdot 10^{1} \cdot \sqrt{10^{80}} \approx 10^{-4}<<1
\end{aligned}
$$

This is assuming that one is having large, but non infinite temperatures which would not be in excess of, say $T \sim 10^{2} \cdot T_{Q G-t h r e s h o l d} \approx 10^{34} \mathrm{~K}$. We are also assuming a base line time unit of $t^{*}<t_{P} \sim 10^{-45} \mathrm{~s}$, and $\delta \cdot t \leq t_{P} \sim 10^{-45} \mathrm{~s}$. Should we have $\delta \cdot t<<t_{P} \sim 10^{-45} s$, then the right hand side of Eqn. 41 above will be much smaller.

So, the nomenclature of 0 put in denotes near present day conditions for a discrete equation model of Eq. (41) leads to Dowker's paper on causal sets which requires the following ordering with a relation $\prec$, where we assume that initial relic space time is replaced by an assembly of discrete elements, so as to create, initially, a partially ordered set $C$ :

(1) If $x \prec y$, and $y \prec z$, then $x \prec z$

(2) If $x \prec y$, and $y \prec x$, then $x=y$ for $x, y \varepsilon C$

(3) For any pair of fixed elements $x$ and $z$ of elements in $C$, the set $\{y \mid x \prec y \prec z\}$ of elements lying in between $\mathrm{x}$ and $\mathrm{z}$ is finite, which is fulfilled by Eq. (44) below.

$$
\left[\frac{a\left(t^{*}+\delta t\right)}{a\left(t^{*}\right)}\right]<1
$$

Items (1) and (2) give us that we have $C$ as a partially ordered set and the third item permits local finiteness. This when combined with as a model for how the universe evolves via a scale factor equation permits violation of partial ordering. It is our contention that this will lead to the increase in entropy as the instanton is broken by Eq. (46) above.

Another way to present this, and to get a tie into chaotic evolution is to make the following approximations to Eq. (40) above: If $u=a^{-1}$

$$
\sqrt{\frac{\Lambda}{3}} \cdot \int d t \equiv-\int \frac{d u}{\sqrt{1+\frac{8 \pi}{\Lambda}\left[\left(\rho_{r e l}\right)_{0} a_{0}^{4} u^{4}+\left(\rho_{m}\right)_{0} a_{0}^{3} u^{3}\right]}}
$$

Integrating leads to the following polynomial expression for $u=a^{-1}$

$$
u^{9}+A_{1} \cdot \frac{u^{8}}{a_{0}}+A_{2} \cdot \frac{u^{7}}{a_{0}^{2}}-A_{3} \cdot\left(\frac{\Lambda}{8 \pi}\right) \cdot \frac{u^{5}}{a_{0}^{4}}-A_{4} \cdot\left(\frac{\Lambda}{8 \pi}\right) \cdot \frac{u^{4}}{a_{0}^{5}}+A_{5} \cdot\left(\frac{\Lambda}{8 \pi}\right)^{2} \cdot \frac{u^{1}}{a_{0}^{8}}+A_{6} \cdot\left(\frac{\Lambda}{8 \pi}\right)^{2} \cdot \frac{t}{a_{0}^{9}} \cong 0
$$

We could go considerably higher in polynomial roots of Eq.(47) above, depending upon the degree of accuracy we wished to obtain. This truncation so picked above is assuming a non infinite value of $u=a^{-1}$, as well as a non zero value, and non infinite value for the $\Lambda$ term. In doing so, we would obtain an extremely non standard evolution for the scale factor, assuming when we do so that

$$
A_{1}=\frac{9}{4} \cdot \frac{\left(\rho_{m}\right)_{0}}{\left(\rho_{\text {rel }}\right)_{0}} \quad A_{2}=\frac{\left(\rho_{m}\right)_{0}^{2}}{\left(\rho_{\text {rel }}\right)_{0}^{2}} \quad A_{3}=\frac{1 / 5}{\left(\rho_{\text {rel }}\right)_{0}^{1}} \quad A_{4}=\frac{\left(\rho_{m}\right)_{0} \cdot(1 / 4)}{\left(\rho_{\text {rel }}\right)_{0}^{2}} \quad A_{5}=\frac{1}{\left(\rho_{\text {rel }}\right)_{0}^{2}} \quad A_{6}=\frac{1}{\left(\rho_{\text {rel }}\right)_{0}^{2}} \cdot \sqrt{\frac{\Lambda}{3}}
$$

Beginning with Mukhanov ${ }^{17}$ setting his spatial dimension for a 'particle' as $\rho_{m} \sim r \propto a \sim \hat{\varepsilon}^{-1}$, we look at how to implement Eq. (46) above. We have that if we write 


$$
a\left(t^{*}+\delta \cdot t\right)<a\left(t^{*}\right) \Leftrightarrow \frac{1}{\Delta \hat{\varepsilon}} \cdot<\frac{1}{\hat{\varepsilon}_{1}} \Leftrightarrow \hat{\varepsilon}_{1}<\Delta \hat{\varepsilon}<\hat{\varepsilon}_{2}
$$

The transition from $\hat{\varepsilon}_{1}=\hat{\varepsilon}_{2}-\Delta \hat{\varepsilon} \longrightarrow \Delta \hat{\varepsilon}$ as $t^{*} \rightarrow t^{*}+\delta \cdot t$. This would correspond to the following picture. Have $\Delta \hat{\varepsilon}$ be the net energy density inside an instanton, with a boundary region of $\hat{\varepsilon}_{2}-\Delta \hat{\varepsilon} \geq 0$ energy density on the boundary of the Instanton. As the $\hat{\varepsilon}_{2}-\Delta \hat{\varepsilon} \rightarrow 0$, we have a release of $\Delta \hat{\varepsilon}$ from the interior of the soliton (instanton). If we look at the following Seth Lloyd ${ }^{8}$ supplied relationship,

i.e. if we set energy density dimensions here as $\rho_{2}$

$$
[\text { \#operations }]_{2} \approx \rho_{2} \cdot(c \equiv 1)^{5} \cdot t_{P}^{4} \leq 10^{120}
$$

\section{Open question. Could a causal discontinuity lead to an explanation of fluctuations in the CMBR radiation spectrum?}

One very valid area of inquiry that will be investigated in the future is the following: Is this argument valid if there is some third choice of set structure (for instance do self referential sets fall into one category or another?). The answer to this we think lies in (entangled?) vortex structure of space time, along the lines of structure similar to that generate in the laboratory by Ruutu 20. Self referential sets may be part of the generated vortex structure, and we will endeavor to find if this can be experimentally investigated. If the causal set argument and its violation via this procedure holds, we have the view that what we see a space time 'drum' effect with the causal discontinuity forming the head of a 'drum' for a region of about $10^{10}$ bits of 'information' before our present universe up to the instant of the big bang itself for a time region less than $t \sim 10^{-44}$ seconds in duration, with a region of increasing bits of 'information' going up to $10^{120}$ due to vortex filament condensed matter style forming through a symmetry breaking phase transition.

The relevance this has with CMBR is two fold. Conventional fluctuations leading to the CMBR angular separation of the particle-horizon distance of about $\Delta \theta \approx 1.4^{\circ}$, and this is in line with acoustic peaks in the WMAP power spectrum starting at about $l \sim 200$ for the multipole moment . Conventional treatment of the CMBR data makes generous use of error bars. Sharkar has raised the specific possibility in his talk ' Cosmology beyond the Standard Model' in ICGC-07, Pune, India, and also in print 21 that there is another explanation as to the error bars, namely that as reported in Sarkar's BadHonnef07 talk 22 that there is a fluctuation in early universe structure, beyond the normal perturbations associated with the standard model which need to be investigated. In particular, Blanco-Pillado et al in 2004 23investigated race track models of inflation where there was investigation of a more complex version of a scalar field evolution equation of the form

$$
\ddot{\phi}^{i}+3 H \dot{\phi}^{i}+\Gamma_{j k}^{i} \dot{\phi}^{j} \dot{\phi}^{k}+g^{i j} \frac{\partial V}{\partial \phi^{j}}=0
$$

This has real and imaginary components to the scalar field which can be identified as of the form $X_{i}$ for the real part to the scalar field $\phi^{i}$, and $Y_{j}$ for the imaginary part of the scalar field $\phi^{j}$, as well as having

$$
H^{2} \equiv\left(\frac{\dot{a}}{a}\right)^{2}=\frac{8 \pi G}{3} \cdot\left[\frac{1}{2} \cdot g^{i j} \dot{\phi}^{i} \dot{\phi}^{j}+V\right]
$$

Blainco-Pillado et al. ${ }^{23}$ use this methodology, using the physics of the christoffel symbol as usually given by

$$
\Gamma_{j k}^{i}=\frac{1}{2} \cdot g^{i, \sigma} \cdot\left(\partial_{j} g_{k, o}+\partial_{k} g_{\rho, j}-\partial_{\rho} g_{j, k}\right)
$$

If one has no coupling of terms as in an expanding universe metric of the form 24

$$
d S^{2}=-d t^{2}+a^{2}(t) \cdot \delta_{j}^{i} d x^{i} d x^{j}
$$

\footnotetext{
20 Ruutu, V., Eltsov, V, Gill, A., Kibble, T., Krusius, M., Makhlin, Y.G., Placais, B., Volvik, G, and Wen, Z., "Vortex Formation in neutron - irradiated 3He as an analog of cosmological defect formation," Nature 382, 334-336 (25 July 1996)

21 Lalak, Z., Ross, G., and Sakar, S., "Racetrack Inflation and assisted Moduli Stabilization", arXIV hep-th / 0503178 v 2, 12 June, 2006 $22 \mathrm{http}: / /$ www-thphys.physics.ox.ac.uk/user/SubirSarkar/

23 Blanco-Pillado, J., Burgess, C., Cline, J., Escoda, C., Gomez - Reino , M., Kallosh, R., Line, A., Quevedo, F., “Racetrack Inflation”, JHEP 0411 (2004) 076, arXIV hep-th / 0411011

24 Carroll, S., Spacetime and Geometry:: An Introduction to General Relativity, Addison Wesley Co., San Francisco, 2005
} 
Then the christoffel symbols take the form given by

$$
\begin{aligned}
& \Gamma_{00}^{i}=0, \\
& \Gamma_{j 0}^{i}=\Gamma_{0 j}^{i}=\frac{\dot{a}}{a} \delta_{j}^{i}, \\
& \Gamma_{j k}^{i}=0
\end{aligned}
$$

The implications for the Eq. (51) value of the scalar evolution equation are that we have

$$
\ddot{\phi}^{i}+3 H \dot{\phi}^{i}+(\dot{a} / a) \cdot \delta_{j}^{i} \dot{\phi}^{j} \dot{\phi}^{0}+g^{i j} \frac{\partial V}{\partial \phi^{j}}=0
$$

If we can write as follows, i.e. say that we have $\dot{\phi}^{0} \sim 0$, as well as have $g^{i j} \equiv g^{i i}= \pm 1$,

$$
\begin{gathered}
g^{00} \equiv 1, g^{i i}=-1, \text { if } i=j \neq 0 \\
\ddot{\phi}^{i}+3 H \dot{\phi}^{i}+H \cdot \dot{\phi}^{i} \dot{\phi}^{0}+g^{i i} \frac{\partial V}{\partial \phi^{i}}=0 \\
\ddot{\phi}^{i}+3 H \dot{\phi}^{i}-\frac{\partial V}{\partial \phi^{i}}=0 \\
\dot{\phi}^{0} \sim 0 \Rightarrow
\end{gathered}
$$

On the other hand,

$$
\dot{\phi}^{0} \sim 1 \Rightarrow \ddot{\phi}^{i}+4 H \dot{\phi}^{i}-\frac{\partial V}{\partial \phi^{i}}=0 \quad \text { provided } t \leq t_{P}
$$

Otherwise, taking into account the causal discontinuity expression, we claim we will be working with

$$
\ddot{\phi}^{i}+3 H \dot{\phi}^{i}-\frac{\partial V}{\partial \phi^{i}}=0 \text { provided } t>t_{P}
$$

For very short time duration, and looking at the case for chaotic inflation, we would be working with, in this situation $\frac{\partial V}{\partial \phi^{i}} \cong M_{P} \phi_{i}$. Set an ansatz with regards to

$$
\begin{array}{r}
\ddot{\phi}^{i}+4 H \dot{\phi}^{i}-M_{P}^{2} \phi_{i}=0 \text { provided } t \leq t_{P} \\
\text { If } \phi_{i} \sim e^{b t} \text {, Eqn. (62) } \Rightarrow b^{2}+4 H b-M_{P}^{2}=0 \Rightarrow b=-2 H \pm \sqrt{4 H^{2}+4 M_{P}^{2}}
\end{array}
$$

This would lead to, if provided $t \leq t_{P}$, and for a short period of time, $\mathrm{H}$ is a constant

$$
\phi \approx c_{1} \exp \left[\left(-2 \cdot H-\sqrt{4 H^{2}+4 M_{P}^{2}}\right) \cdot t\right\rfloor+c_{2} \exp \left[\left(-2 H+\sqrt{4 H^{2}+4 M_{P}^{2}}\right) \cdot t\right]
$$

Similarly, for $t>t_{P}$, assuming for a short period of time that $\mathrm{H}$ is approximately a constant.

$$
\phi \approx c_{1} \exp \left[\left(-\frac{3}{2} \cdot H-\sqrt{\frac{9}{4} H^{2}+4 M_{P}^{2}}\right) \cdot t\right]+c_{2} \exp \left[\left(-\frac{3}{2} H+\sqrt{\frac{9}{4} H^{2}+4 M_{P}^{2}}\right) \cdot t\right]
$$

Ups shot is that for $t>t_{P}$, there is a greater rate of growth in the $\phi$ scalar field than is the case when $t \leq t_{P}$

How to tie in the entropy with the growth of the scale function?

Racetrack models of inflation, assuming far more detail than what is given in this simplistic treatment provide a power spectrum for the scalar field given by

$$
P \sim \frac{1}{150 \pi^{2}} \cdot \frac{V(\phi)}{\epsilon}
$$


This is assuming a slow roll parameter treatment with $\in<<1$, and for $t>t_{p}$. Eq. (65) would be growing fairly rapidly in line with what is said about Eq. (64) above. An increase in scalar power, is then proportional to an increase in entropy via

$$
\left|\frac{\Delta E}{l_{P}^{3}}\right| \sim\left|\frac{\Delta P \in 150 \pi^{2}}{l_{P}^{3}}\right| \approx|\Delta S|
$$

Now, how does this tie in with the lumpiness seen in the CMBR spectra? In an e mail communication, Sarkar summarized the situation up as follows 25 :

"Quasi-DeSitter spacetime during inflation has no "lumpiness" - it is necessarily very smooth. Nevertheless one can generate structure in the spectrum of quantum fluctuations originating from inflation by disturbing the slow-roll of the inflaton - in our model this happens because other fields to which the inflaton couples through gravity undergo symmetry breaking phase transitions as the universe cools during inflation"

If we use what is in Appendix II, namely the non flat space generalization of the flat space De Alembertian leading to, for a quartic potential as given in Appendix II

and generalizations of

$$
\begin{aligned}
& \phi^{2}=\frac{1}{\widetilde{a}} \cdot\left\{c_{1}^{2}-\left[\alpha^{2}+\frac{\kappa}{6 a^{2}(t)}+\left(M(T) \approx \varepsilon^{+}\right)\right]\right\} \\
& \underset{M(T \sim h i g h) \rightarrow 0}{\longrightarrow} \phi^{2} \neq 0
\end{aligned}
$$

$$
\begin{aligned}
& \phi^{2}=\frac{1}{\widetilde{a}} \cdot\left\{c_{1}^{2}-\left[\alpha^{2}+\frac{\kappa}{6 a^{2}(t)}+\left(M(T) \neq \varepsilon^{+}\right)\right]\right\} \\
& \underset{M(T \sim L o w) \neq 0}{\longrightarrow} \phi^{2} \approx 0
\end{aligned}
$$

The mass being referred to fades out if there is a temperature increase. So happens that there is one. And this due to the worm hole transfer of thermal heat and the like from a prior universe. This is done and can be made far more complex if the De Alembertian has off diagonal terms in it

i.e. if one does not insist upon simple Euclidian space, the Laplacian takes the form ${ }^{24}$

$$
\Delta \phi \equiv \eta^{u, v} \partial_{u} \partial_{v} \phi \equiv \partial_{i} \partial^{i} \phi+\partial^{i} \phi \cdot \partial_{i} \ln \sqrt{|g|}
$$

We claim that generalization for Eq. (67) and Eq. (68) will lead in the case of cooling for a scalar field system in the aftermath of immediate rapid expansion of the scalar field a very different, and far more complicated dynamic than is given by Eq. (68)

Recall what is given in modeling the pure Dilatonic potential, i.e. as given by Lalak, Ross, and Sakar ${ }^{21}(2006)$. This potential has a minimum if $\mathrm{B} / \mathrm{A}>1$ where it can vanish, and it has a non zero minimum if we set $1>\mathrm{B} / \mathrm{A}>N_{2} / N_{1}$

$$
\begin{aligned}
& V(s, \phi)=\frac{1}{2 s} \cdot\left(A \cdot\left(2 s+N_{1}\right) \cdot e^{-s / N_{1}}+B \cdot\left(2 s+N_{2}\right) \cdot e^{-s / N_{2}}\right)^{2} \\
& +\frac{1}{s} \cdot A \cdot B \cdot\left(2 s+N_{1}\right) \cdot\left(2 s+N_{2}\right) \cdot e^{-s / N_{1}} \cdot e^{-s / N_{2}} \cdot(1-\cos (\phi \cdot \varepsilon))
\end{aligned}
$$

This is assuming that we are having $s \rightarrow N_{a} \neq \infty$, leading to minima for $\phi_{k}=k \pi / \varepsilon$, with $\mathrm{k}$ being the positive and negative integers, i.e. this helps delineate between two condensates. If we have a complex scalar field $\phi_{j}=X_{j}+i \cdot Y_{j}$. we have moduli arguments which add far more structure, i.e. we are getting into Calabi-Yau compactification issues. Appendix III offers a simpler potential system. But that system plus Eq. (70) must have spectral index behavior, i.e. reflecting inflation and the early universe, which matches WMAP data.

Point which is to be made here, is that the richer the structure with respect to Eq. (70), and its race track version which has real and imaginary components to a scalar field, the less tenable the simple Eq. (67) and Eq. (68) pictures of simply rising and falling scalar potentials are. So the following claim is made.

CLAIM 1: In the initial phase of expansion in an inflationary sense, the period of time $t<t_{P}$ corresponds with a scalar field given by Eq. (67) and Eq. (68). As we have a rapidly increasing temperature, we have no complexity of the sort implied by Eq. (70) above

CLAIM 2 : In the cool down period before the re heating period after inflation, we have additional structure put in, enough so , so that multiple minima and fluctuations exists which would give far more definition as to local scalar power spectra. I.e. 
we are looking at

$$
\left|\frac{\Delta V(s, \phi)}{l_{P}^{3}}\right| \sim\left|\frac{\Delta P \in 150 \pi^{2}}{l_{P}^{3}}\right| \approx|\Delta S| \Leftrightarrow|\Delta P| \sim\left|\frac{\Delta V(s, \phi)}{\in 150 \pi^{2}}\right|
$$

Provided that we have non zero minimum if we set $1>\mathrm{B} / \mathrm{A}>N_{2} / N_{1}$ for $\Delta V$, we claim that then we are having the basis for non zero fluctuations seen as given in Sarkar's Bad Honnef 07 portrayal of CMBR.

We can use the criteria of Appendix IV, which gives realistic data input parameters as to the variance of the CMBR spectra. In particular, we can take Eq. (3) of Appendix IV and splicing that in on a new derivation as to $C_{l}$ power spectra. I.e. $C_{l}$ of Appendix $\mathbf{V}$ is an incredibly crude model, which depends upon Eq. (3) of that section for a power law, which then leads to how to re construct, assuming NO time dependence upon the Hubble Parameter; i.e. $\dot{H}=0$, to come up with a tensor type of expression for $C^{(T)}$ based upon what can be called very naïve assumptions.

Here we can make the following assertion. Which I think is entirely reasonable. Especially with regards to

Gravitational waves. This is from Durrer, 26 and is a foundation for additional work which can be done i.e.

$$
C_{l}^{(T)}=\frac{2}{\pi} \cdot \int d k k^{2}\left\langle\left|\int_{\eta \text { dec }}^{\eta_{0}} d \eta \cdot \dot{H}(\eta, k) \cdot \frac{j_{l}\left(k\left(\eta_{0}-\eta\right)\right)}{\left(k\left(\eta_{0}-\eta\right)\right)^{2}}\right|^{2}\right\rangle \cdot \frac{(l+2) !}{(l-2) !}
$$

We can appeal to simplified models as to how to come up with $\dot{H}$. First of all, consider the causal discontinuity equation argument. This is one phase as to implementation, i.e. look at $\dot{H}=\frac{d}{d \eta}\left(\frac{\dot{a}}{a}\right)$. This is where we are working directly with Eq. (52) in part, and at the regime of at least partial causal discontinuity, we are working with Eq. (41) The interplay between these two equations in part can lead to an effective re construction of a potential system, which in part should in its structure, have some similarities with the race track potential. Appendix VI also gives guidance as to re construction of the potential system we can work with, and also compare it with the different race track models so outlined.

In addition to this treatment of how to get a CMBR reconstruction of gravitational tensor fluctuations, we can also look at observational efforts to confirm, or falsify different models of $\left|\frac{\Delta P \in 150 \pi^{2}}{l_{P}^{3}}\right| \approx|\Delta S|$, i.e. how the entropy varies will be in its own way will affect the power spectra, which in turn affects confirming or falsifying the spectral index $n_{s}=1-\frac{d \ln P}{d N} \approx .95 \pm .02$. Here, $\mathrm{N}$ is the number of e folding in inflation and we can follow through on elementary calculations of how $P$ varies due to choices of potential system we are examining. I.e. recall Sarkar's 2001 investigation of a simple choice of variant of the standard chaotic inflationary potential given by 27

$$
V \equiv V_{0}-c_{3} \phi^{3}+\frac{1}{2} \lambda \cdot \phi^{2} \cdot \rho^{2}+\ldots
$$
Sarkar treated the inflaton as having a varying effective mass, with an initial value of effective mass of $m_{\phi}{ }^{2}=\frac{d^{2} V}{d \phi^{2}}$ given
a before and after phase transition value of

$$
m_{\phi}^{2}=-\left.6 c_{3} \cdot\langle\phi\rangle\right|_{\text {Before-phase-transition }} \underset{\text { phase-transition }}{\longrightarrow}-6 c_{3} \cdot\langle\phi\rangle+\left.\lambda \cdot \Sigma^{2}\right|_{\text {after-phase-transition }}
$$

This is, when Sarkar did it, with $\lambda=\kappa \cdot m^{2} / M_{P}^{2}$ as a coupling term. This would also affect the spectral index value, and it also would be a way to consider an increase in inflation based entropy. The only draw back to this phenomenological treatment is that it in itself does not address the formation of an instanton in the very beginning of inflation, a serious draw back since this does not also give an entry into the formation of the layers of complexity which we think is more accurately reflected in the transferal of state from a growing value of the magnitude of the scalar field as given by Eq. (67) and Eq. (68) as temperature flux flows in from a prior universe, to the cooling off period we think is necessary for the formation of a complex scalar field and its analogies in the race track style models, as in Eq (70), and Appendix II below. Eq. (72) with its treatment of tensorial contributions to the CMBR has its counter part, an implied release in relic gravitons which may, or may not be amendable to observational techniques. We would most likely imply their existence indirectly via use of Eq. (72)

26 Durrer, R., "Cosmological Pertubation theory", From the book "Physics of the Early Universe", in Lecture Notes in Physics, 653", with Papantonopoulos, E., as editor and printed by Springer- Verlag 2004

27 Hunt, P. and Sakar, S., "Multiple Inflation and the WMAP "glitches” “, Phys Rev D Volume 70, 103518 
and seeing if they can be linked to the behavior of the inflaton generating a new burst of entropy at the onset of inflation. Appendix VII shows what we may wish to consider as to relic graviton production which is linkable to the worm hole, and causal discontinuity discussion we have brought up, with regards to early universe entropy generation. We also will make reference that this has been linked to brane theory via Appendix VIII material.

\section{Conclusion. Match up with Smoot's table}

In a colloquium presentation done by Smoot in Paris ${ }^{5}$ (2007); he alluded to the following information theory constructions which bear consideration as to how much is transferred between a prior to the present universe in terms of information 'bits'.

0 ) Physically observable bits of information possibly in present Universe - $10^{180}$

1) Holographic principle allowed states in the evolution / development of the Universe - $10^{120}$

2) Initially available states given to us to work with at the onset of the inflationary era- $10^{10}$

3) Observable bits of information present due to quantum / statistical fluctuations $-10^{8}$

Our guess is as follows. That the thermal flux so implied by the existence of a worm hole accounts for perhaps $10^{10}$ bits of information. These could be transferred via a worm hole solution from a prior universe to our present, and that there could be , perhaps $10^{120}$ minus $10^{10}$ bytes of information temporarily suppressed during the initial bozonification phase of matter right at the onset of the big bang itself .

'Then after the degrees of freedom dramatically drops during the beginning of the descent of temperature from about $T \approx 10^{32}$ Kelvin to at least three orders of magnitude less, as we move out from an initial red shift

$$
z \approx 10^{25}
$$

to 28

$$
T \approx \sqrt{\varepsilon_{V}} \times 10^{28} \text { Kelvin } \sim T_{\text {Hawkings }} \cong \frac{\hbar \cdot H_{\text {initial }}}{2 \pi \cdot k_{B}}
$$

Whichever model we can come up with that does this is the one we need to follow, experimentally. And it gives us hope in confirming if or not we can eventually analyze the growth of structure in the initial phases of quantum nucleation of emergent space time29. We also need to consider the datum so referenced as to the irregularities as to the cooling down phase of inflation, as mentioned by Sakar, which will be put in once again ${ }^{25}$

"Quasi-DeSitter spacetime during inflation has no "lumpiness" - it is necessarily very smooth. Nevertheless one can generate structure in the spectrum of quantum fluctuations originating from inflation by disturbing the slow-roll of the inflaton - in our model this happens because other fields to which the inflaton couples through gravity undergo symmetry breaking phase transitions as the universe cools during inflation"

The race track models, after the inflaton begins to decline would be ideal in getting the couplings, and the symmetry breaking. We will refer to this topic in a future publication. We can make a few observations though about the coupling so assumed. First, there is a question of if or not there is a finite or infinite fifth dimension. String theorists have argued for a brane-world with a warped, infinite extra dimension allowing for the inflaton to decay into the bulk so that after inflation, the effective dark energy disappears from our brane. This is achieved by shifting away the decay products into the infinity of the 5th dimension.30 Nice hypothesis, but it presumes CMB density perturbations could have their origin in the decay of a MSSM flat direction. It would reduce the dynamics of the inflaton to be separation between a $D p$ brane and $\bar{D} p$ anti brane via a moduli argument.

What if we do not have an infinite fifth dimension? What if it is compactified only? We then have to change our analysis.

Another thing. We place limits on inflationary models; for example, a minimally coupled $\lambda \phi^{4}$ is disfavored at more than $3 \sigma$. Result? Forget quartic inflationary fields, as has been show by Peiris, Hingshaw et al31. We can realistically hope that WMAP will be able to parse through the race track models to distinguish between the different candidates. So far "First-Year Wilkinson Microwave Anisotropy Probe (WMAP)1 Observations: Implications For Inflation", is giving chaotic inflation a run for its money. We shall endeavor for numerical work using some of the tools brought up in this present discussion for falsifying or confirming the figures 1 and 2 of this text which show variance in the CMBR spectrum. In doing so, we set the stage for showing how Brane-anti brane models of entropy fare with regards to their more classical counter parts.

28 Sanchez, N. 11th Paris Cosmology Colloquium, August 18th, 2007 with respect to Sanchez, N., "Understanding Inflation and Dark Energy in the Standard Model of the Universe", 'D.Chalonge' school

29 Ruutu, V., Eltsov, V, Gill, A., Kibble, T., Krusius, M., Makhlin, Y.G., Placais, B., Volvik, G, and Wen, Z., "Vortex Formation in neutron - irradiated 3He as an analog of cosmological defect formation," Nature 382, 334-336 (25 July 1996)

30 Enqvist, K., Mazumdar, A., Perez--Lorenzana, A., “ Dumping inflaton energy out of this world”,Phys Rev D, Volume 70, 103508

31 H. V. Peiris, H., Komatsu, E., Verde, L., Spergel, D., Bennett, C., Halpern, M., Hinshaw, G., Jarosik, N., Kogut, A., Limon, M., Meyer, S., Page, L., Tucker, G., Wollack, E., and Wright, E.," First-Year Wilkinson Microwave Anisotropy Probe (WMAP) Observations: Implications For Inflation", The Astrophysical Journal Supplement Series, 148:213-231, 2003 September 
The excess $\chi^{2}$ comes mostly from the outliers in the TT spectrum

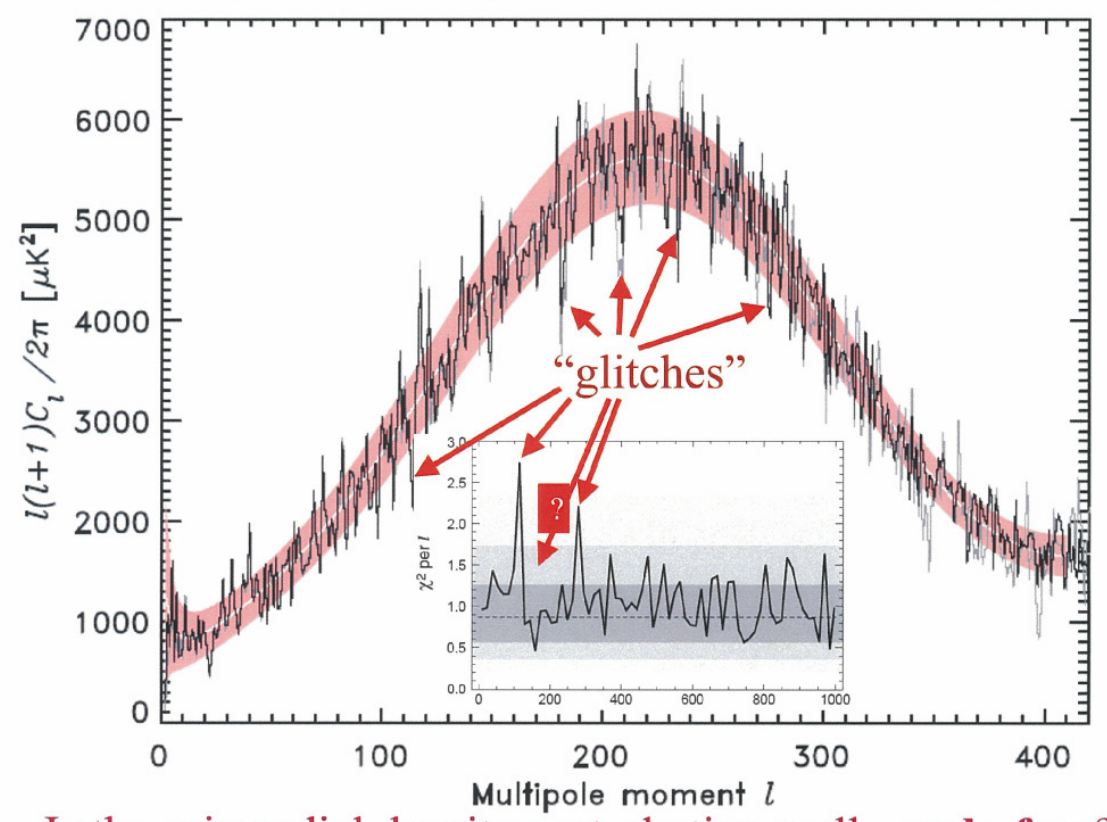

Is the primordial density perturbation really scale-free?

Figure 1. as given by Subir Sarkar, from his web site. Copied from Sarkar's Bad Honnif 07 talk and re produced here with explicit permission of the original presenter. Shows the glitches which need to be addressed in order to make a CMBR data set congruent with an extension of the standard model of cosmology.

In fact the 'power-law $\triangle \mathrm{CDM}$ model' does not fit $W M A P$ data very well

Best-fit: $\Omega_{\mathrm{m}} h^{2}=0.13 \pm 0.01, \Omega_{\mathrm{b}} h^{2}=0.022 \pm 0.001, h=0.73 \pm 0.05, n=0.95 \pm 0.02$

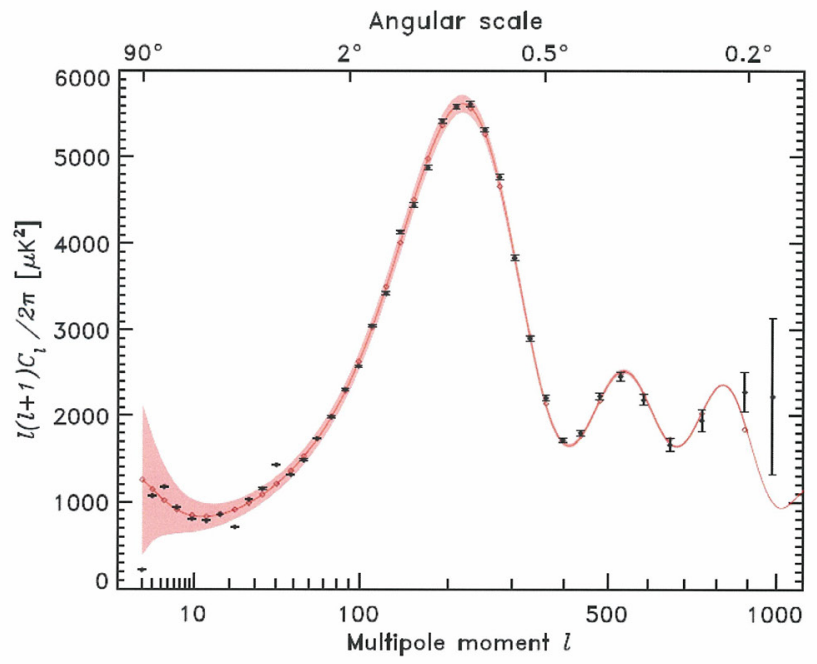

But the $\chi^{2} /$ dof $=1049 / 982 \Rightarrow$ probability of only $\sim 7 \%$ that this model is correct!

Figure 2. Self explanatory. From Sarkar's Bad Honnif07 talk . Reproduced here with permission of Sakar in e mail communication

\section{Appendix I. Details as to forming Crowell's time dependent Wheeler De Witt equation, and its links to Worm holes}

We will fill in the details inherent in Eq. (26) above in the main text.. This will be to show some things about the worm hole we assert the instanton traverses en route to our present universe. Eq (26) of the main text actually comes from the following version of the Wheeler De Witt equation with a pseudo time component added. From Crowell ${ }^{14}$ 


$$
-\frac{1}{\eta r} \frac{\partial^{2} \Psi}{\partial r^{2}}+\frac{1}{\eta r^{2}} \cdot \frac{\partial \Psi}{\partial r}+r R^{(3)} \Psi=(r \eta \phi-r \ddot{\phi}) \cdot \Psi
$$

This has when we do it $\phi \approx \cos (\omega \cdot t)$, and frequently $R^{(3)} \approx$ constant, so then we can consider

$$
\phi \cong \int_{0}^{\infty} d \omega\left[a(\omega) \cdot e^{i k_{\varpi} x^{\mu}}-a^{+}(\omega) \cdot e^{-i k_{\varpi} x^{\mu}}\right]
$$

In order to do this, we can write out the following with regards to the solutions to Eq. (1) put up above.

$$
\begin{aligned}
& C_{1}=\eta^{2} \cdot\left(4 \cdot \sqrt{\pi} \cdot \frac{t}{2 \omega^{5}} \cdot J_{1}(\omega \cdot r)+\frac{4}{\omega^{5}} \cdot \sin (\omega \cdot r)+(\omega \cdot r) \cdot \cos (\omega \cdot r)\right) \\
& +\frac{15}{\omega^{5}} \cos (\omega \cdot r)-\frac{6}{\omega^{5}} \operatorname{Si}(\omega \cdot r)
\end{aligned}
$$

And

$$
C_{2}=\frac{3}{2 \cdot \omega^{4}} \cdot(1-\cos (\omega \cdot r))-4 e^{-\omega \cdot r}+\frac{6}{\omega^{4}} \cdot C i(\omega \cdot r)
$$

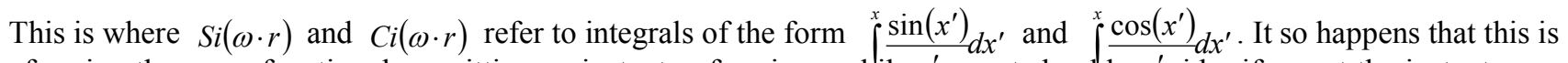
for forming the wave functional permitting an instanton forming, while we next should cornsider if or not the instanton so farmed is stable under evolution of space time leading up to inflation. We argue here that we are forming an instanton whose thermal energy is focused into a wave functional which is in the throat of the worm hole up to a thermal discontinuity barrier at the onset, and beginning of the inflationary era.

\section{Appendix II: The D'Albembertain operation in an equation of motion for emergent scalar fields}

We begin with the D'Albertain operator as part of an equation of motion for an emergent scalar field. We refer to the Penrose potential ( with an initial assumption of Euclidian flat space for computational simplicity) to account for, in a high temperature regime an emergent non zero value for the scalar field $\phi$ due to a zero effective mass, at high temperatures. 32

When the mass approaches far lower values, it, a non zero scalar field re appears.

Leading to $\phi \underset{T \rightarrow 2.7^{0} \text { Kelvin }}{\longrightarrow} \varepsilon^{+} \approx 0^{+}$as a vanishingly small contribution to cosmological evolution

Let us now begin to initiate how to model the Penrose quintessence scalar field evolution equation. To begin, look at the flat space version of the evolution equation

$$
\ddot{\phi}-\nabla^{2} \phi+\frac{\partial V}{\partial \phi}=0
$$

This is, in the Friedman - Walker metric using the following as a potential system to work with, namely:

$$
\begin{aligned}
& V(\phi) \sim-\left[\frac{1}{2} \cdot\left(M(T)+\frac{\Re}{6}\right) \phi^{2}+\frac{\widetilde{a}}{4} \phi^{4}\right] \equiv \\
& -\left[\frac{1}{2} \cdot\left(M(T)+\frac{\kappa}{6 a^{2}(t)}\right) \phi^{2}+\frac{\widetilde{a}}{4} \phi^{4}\right]
\end{aligned}
$$

This is pre supposing $\kappa \equiv \pm 1,0$, that one is picking a curvature signature which is compatible with an open universe. That means $\kappa=-1,0$ as possibilities. So we will look at the $\kappa=-1,0$ values. We begin with. 


$$
\begin{aligned}
& \ddot{\phi}-\nabla^{2} \phi+\frac{\partial V}{\partial \phi}=0 \Rightarrow \\
& \phi^{2}=\frac{1}{\widetilde{a}} \cdot\left\{c_{1}^{2}-\left[\alpha^{2}+\frac{\kappa}{6 a^{2}(t)}+M(T)\right]\right\} \\
& \Leftrightarrow \phi \equiv e^{-\alpha \cdot r} \exp \left(c_{1} t\right)
\end{aligned}
$$

We find the following as far as basic phenomenology, namely

$$
\begin{aligned}
& \phi^{2}=\frac{1}{\widetilde{a}} \cdot\left\{c_{1}^{2}-\left[\alpha^{2}+\frac{\kappa}{6 a^{2}(t)}+\left(M(T) \approx \varepsilon^{+}\right)\right]\right\} \\
& \underset{M(T \sim \text { high }) \rightarrow 0}{\longrightarrow} \phi^{2} \neq 0 \\
& \phi^{2}=\frac{1}{\widetilde{a}} \cdot\left\{c_{1}^{2}-\left[\alpha^{2}+\frac{\kappa}{6 a^{2}(t)}+\left(M(T) \neq \varepsilon^{+}\right)\right]\right\} \\
& \underset{M(T \sim \text { Low }) \neq 0}{\longrightarrow} \phi^{2} \approx 0
\end{aligned}
$$

The difference is due to the behavior of $M(T)$. We use $M(T) \sim$ axion mass $m_{a}(T)$ in asymptotic limits with

$$
m_{a}(T) \cong 0.1 \cdot m_{a}(T=0) \cdot\left(\Lambda_{Q C D} / T\right)^{3.7}
$$

\section{Appendix III: Managing what to do with racetrack inflation, as cool down from initial expansion commences}

P. Brax, A. Davis et al devised a way to describe racetrack inflation as a way to look at how super gravity directly simplifies implementing how one can have inflation with only three $\mathrm{T}$ (scalar) fields. The benefit to what we work with is that we may obtain two gaugino condensates and look at inflation with a potential given by 33

$$
V=V_{0}+V_{1} \cos (a Y)+V_{2} \cos (b Y)+V_{3} \cos (|a-b| \cdot Y)
$$

This has scalar fields $X, \phi$ as relatively constant and we can look at an effective kinetic energy term along the lines of

$$
\mathfrak{J}_{\text {Kinetic }}=3 \cdot(\partial Y)^{2} / 4(\partial X)^{2}
$$

This ultra simple version of the race track potential is chosen so that the following conditions may be applied

(1) Exist a minimum at $Y=Y_{0}$; i.e. we have $V^{\prime}\left(Y_{0}\right)=0$, and $V^{\prime \prime}\left(Y_{0}\right)>0$, when we are not considering scalar fields $X, \phi$

(2) We set a cosmological constant equal to zero with $V\left(Y_{0}\right)=0$

(3) We have a flat saddle at $Y \approx 0$; i.e. $V^{\prime \prime}(0)=0$

(4) We re - scale the potential via $V \rightarrow \lambda V$ so as to get the observed power spectra $P=4 \times 10^{-10}$

Doing all this though frequently leads to the odd situation that $|a-b|$ must be small so that $X>>1$ in a race track potential system when we analyze how to fit Eq. (1) for flat potential behavior modeling inflation. This assumes that we are working with a spectra index of the form so that if the scalar field power spectrum is

$$
P=\frac{V}{150 \pi^{2} \varepsilon}
$$

Then the spectral index of the inflaton is consistent with WMAP data. I.e. if we have the number of e foldings $N>N_{*} \approx .55$

33 Brax, Ph., Davis, A., Davis, S., Jeannerot, R., Postman, M., “ D-term Uplifted Racetrack Inflation”, Journal of Cosmology and Astroparticle Physics, JCAP01(2008) 08 


$$
n_{s}=1-\frac{d \ln P}{d N} \approx .95 \pm .02
$$

These sort of restrictions on the spectral index will start to help us retrieve information as to possible inflation models which may be congruent with at least one layer of WMAP data. This model says nothing about if or not the model starts to fit in the data issues Subir Sarkar identified in is Pune, India lecture in 2007.

\section{Appendix IV. Basic physics of achieving minimum precision in CMBR power spectra measurements}

Begin first of all looking at

$$
\frac{\Delta T}{T} \equiv \sum_{l, m} a_{l m} Y_{l, m}(\theta, \phi)
$$

This leads to consider what to do with

$$
C_{l}=\left\langle\left|a_{l, m}\right|^{2}\right\rangle
$$

Samtleben et al 34consider then what the experimental variance in this power spectrum, to the tune of an achievable precision given by

$$
\frac{\Delta C_{l}}{C_{l}}=\sqrt{\frac{2}{2 l+1}} \cdot\left(\frac{1}{\sqrt{f_{s k y}}}+\frac{4 \pi \cdot\left(\Delta T_{\text {exp }}\right)^{2}}{C_{l}} \cdot \sqrt{f_{s k y}} \cdot e^{l^{2} \sigma_{b}^{2}}\right)
$$

$f_{s k y}$ is the fraction of the sky covered in the measurement, and $\Delta T_{\text {exp }}$ is a measurement of the total experimental sensitivity of the apparatus used. Also $\sigma_{b}$ is the width of a beam, while we have a minimum value of $l_{\min } \approx(1 / \Delta \Theta)$ which is one over the fluctuation of the angular extent of the experimental survey.

I.e. contributions to $C_{l}$ uncertainty from sample variance is equal to contributions to $C_{l}$ uncertainty from noise. The end result is

$$
4 \pi \cdot f_{s k y}=C_{l} \cdot\left(\exp \left[-l^{2} \sigma^{2}\right]\right) /(\Delta T)^{2}
$$

\section{Appendix V : Cosmological perturbation theory and tensor fluctuations (Gravity waves)}

Durrer reviews how to interpret $C_{l}$ in the region where we have $2<l<100$, roughly in the region of the Sachs-Wolf contributions due to gravity waves. We begin first of all by looking at an initial perturbation, using a scalar field treatment of the 'Bardeen potential' $\Psi$ This can lead us to put up, if $H_{i}$ is the initial value of the Hubble expansion parameter

$$
k^{3}|\Psi|^{2} \cong\left(\frac{H_{i}}{M_{P}}\right)^{2}
$$

And

$$
\left\langle|\Psi|^{2}\right\rangle \cdot k^{3}=A^{2} k^{n-1} \cdot \eta_{0}^{n-1}
$$

Here we are interpreting $A=$ amplitude of metric perturbations at horizon scale, and we set $k=1 / \eta_{0}$, where $\eta$ is the conformal time, according to $d t \equiv a d \eta=$ physical time, where we have $a$ as the scale factor.

Then for $2<l<100$, and $-3<n<3$, and a pure power law given by

34 Samtleben, D., Staggs, S., Winstein, B., "The Cosmic Microwave Background for Pedestrians: A Review for Particle and Nuclear Physicists", Annu..Rev . Nucl. Part. Sci. 2007, 57, : 245-83 


$$
\left\langle|H(k, \eta=1 / k)|^{2}\right\rangle \cdot k^{3}=A_{T}^{2} k^{n_{T}} \cdot \eta_{0}^{-n_{T}}
$$

We get for tensor fluctuation, i.e. gravity waves, and a scale invariant spectrum with $n_{T}=0$

$$
C_{l}^{(T)} \approx \frac{A_{T}^{2}}{(l+3) \cdot(l-2)} \cdot \frac{1}{15 \pi}
$$

\section{Appendix VI . Formation of the Scalar Field, Bifurcation Results}

Start with Padamans's formulas ${ }^{12}$ :

$$
\begin{gathered}
V(t) \equiv V(\phi) \sim \frac{3 H^{2}}{8 \pi G} \cdot\left(1+\frac{\dot{H}}{3 H^{2}}\right) \\
\phi(t) \sim \int d t \cdot \sqrt{\frac{-\dot{H}}{4 \pi G}}
\end{gathered}
$$

If $H=\dot{a} / a$ is a constant, Eq. (2) gives us zero scalar field values at the beginning of quantum nucleation of a universe. At the point of accelerated expansion (due to the final value of the cosmological constant), it also gives an accelerating value of the cosmological scale-factor expansion rate._We justify this statement by using early-universe expansion models, which have $a\left(t_{\text {INITILL }}\right) \sim e^{H \cdot t}$. This leads to the derivative of $H=\dot{a} / a$ going to zero. This is similar to present-time development of the scalar factor along the lines of $a\left(t_{\text {later }}\right) \sim e^{(\Lambda[\text { present-day } t)}$, also leading to the derivative of $H=\dot{a} / a$ going to zero. When both situations occur, we have the scale factor $\phi=0$. Between initial and later times, the scale factor no longer has exponential time dependence, due to it growing far more slowly, leading to $\phi \neq 0$.

Both regimes as specified by Eq. (2) above lead to zero values for a quintessence scalar field. But it does not stop there. We will show later that in actuality, the scalar field likely damps out far before the CMBR barrier value of expansion when $Z=1100$, about 380,000 to 400,000 years after the big bang.

\section{Claim 1: We Observe that the Scalar Field $\phi(t)$ Is Zero at the Onset of the Big Bang, and Also Is Zero during the Present Cosmological Era}

This scalar "quintessence" field is non zero in a brief period of time right after the inflationary era."

We show this by noting that in Eq. (2), the time derivative of $H=\dot{a} / a$ goes to zero when both the scale factors $a\left(t_{\text {INITIAL }}\right) \sim e^{H \cdot t \text { (initial) }}$, and $a\left(t_{\text {later }}\right) \sim e^{\Lambda[\text { present-day }] \cdot(\text { later })}$. The exponential scale factors in both cases (the initial inflationary environment and the present era) lead to the time derivative of the $H=\dot{a} / a$ expression in Eq. (2) going to zero.

Sub point to claim 1: The existence of two zero values of the scalar field $\phi(t)$ at both the onset and at a later time implies a bifurcation behavior for modeling quintessence scalar fields. This is due to the non- zero $\phi(t)$ values right after the initiation of inflation.

\section{Appendix VII : Open questions as to what the large vacuum energy implies for initial conditions for graviton production, plus graviton production in a relic setting}

If we have a non infinite but huge negative value of the cosmological vacuum energy in the wormhole, then we have $10^{10}$ bits of computing information. When we leave the wormhole, we have $10^{120}$ bits of computing information We specify a transition between the two regions in terms of a causal discontinuity regime created by a(t) chaotic behavior due initially to the initially very large value of thermal vacuum energy transmitted.

Details, and many more of them are needed to bridge this transition to the problem of structure formation and a drop of temperature. If we look at Ruutu's (1996) ground breaking experiment we see vortex line filaments rapidly forming. Here are 
a few open questions which should be asked.

1) Do the filaments in any shape or form have an analogy to the cosmic strings so hypothesized by String theorists ? My guess is a flat MAYBE but one cannot be certain of this. This deserves to be analyzed fully. If they have an analogy to cosmic strings, then what is the phase transition from a maximally entangled space time continuum, with a soliton type behavior for temperatures of the order of $T \sim 10^{32} \mathrm{Kelvin}$ to the formation of these stringy structures.

2) What is the mechanism for the actual transition from the initial 'soliton' at high temperatures to the symmetry breaking phase transition? This is trickier than people think. Many theorists consider that, in tandem with Ruutu's (1996) experiment that Axion super partners, Saxions, actually are heated up and decay to release entropy. Do we have structures in initial space time analogous to super fluids allowing us to come up with such a transformation. Do axions/ Saxion super partner pairs exist in the onset of thermal transition from a prior universe to our present universe? How could this be experimentally determined with rigorous falsifiable experimental analysis?

3) One of the models considered as a super fluid candidate for this model has been the di quark one. This however was advanced by Zhitinisky 35(2002) in terms of 'cold dark matter'. Could some analogy to di quarks be used for initial states of matter thermally impacted by a transfer of thermal energy via a wormhole to form a cosmic 'bubble' in line with the initial plasma state given in Ruutu's (1996) experiment?

4) Do the formation of such initial conditions permit us to allow optimal conditions for graviton production? If so, can this be transferred to engineering prototypes? How can this be modeled appropriately?

Here is a very simplified model as to what we may be able to expect if there is actual relic graviton production. I.e. Detecting gravitons as spin 2 objects with available technology. To briefly review what we can say now about standard graviton detection schemes, Rothman 36 states that the Dyson seriously doubts we will be able to detect gravitons via present detector technology. The conundrum is that if one defines the criterion for observing a graviton as

$$
\frac{f_{\gamma} \cdot \sigma}{4 \cdot \pi} \cdot\left(\frac{\alpha}{\alpha_{g}}\right)^{3 / 2} \cdot \frac{M_{s}}{R^{2}} \cdot \frac{1}{\varepsilon_{\gamma}} \geq 1
$$

Here,

$$
f_{\gamma}=\frac{L_{\gamma}}{L}
$$

This has $\frac{L_{\gamma}}{L}$ a graviton sources luminosity divided by total luminosity and $R$ as the distance from the graviton source, to a detector. Furthermore, $\quad \alpha=e^{2} / \hbar$ and $\alpha_{g}=G m_{p}^{2} / \hbar$ a constants $\mathrm{r}$, while $\varepsilon_{\gamma}$ is the graviton P.E. As stated in the manuscript, the problem then becomes determining a cross section $\sigma$ for a graviton production process and $f_{\gamma}=\frac{L_{\gamma}}{L}$.

If this is the case, then what can we do to see how relic gravitons may emerge if we have a worm hole transferred burst of thermal/ vacuum energy? 37

35 Zhitinisky, A., "Dark Matter as Dense Color Superconductor' arXIV: astro-ph/0204218 v1 12 April 2002. Refer to the last version of this document 36 Rothman, T. and Boughn, S., "Can Gravitons be Detected ?", Foundations of Physics, 36, No. 12," 1801-1825 (December 2006), arXIV gr-qc/0601043 v 202 Jan 2006

37 Beckwith, A.W, "How can Brane World physics influences the formation of a cosmological constant relevant to graviton production?" arXIV physics/0612010, accessible as http://arxiv.org/abs/physics/0612010, E.J T.P., 4, No. 15, 105-142 (July 2007a). 
Table 1. With respect to phenomenology

\begin{tabular}{|c|c|c|c|}
\hline Time & Thermal inputs & Dynamics of axion & Graviton Eqn. \\
\hline Time $0 \leq t<<t_{P}$ & $\begin{array}{l}\text { Use of quantum gravity to give } \\
\text { thermal input via quantum } \\
\text { bounce from prior universe } \\
\text { collapse to singularity. Brane } \\
\text { theory predicts beginning of } \\
\text { graviton production. }\end{array}$ & $\begin{array}{c}\text { Axion wall dominant feature of } \\
\text { pre inflation conditions, due to } \\
\text { Jeans inequality with enhanced } \\
\text { gravitational field, } \\
\text { Quintessence scalar equation of } \\
\text { motion valid for short time } \\
\text { interval }\end{array}$ & $\begin{array}{l}\text { Wheeler formula for relic } \\
\text { graviton production beginning } \\
\text { to produce gravitons due to } \\
\text { sharp rise in temperatures. }\end{array}$ \\
\hline Time $0 \leq t<t_{P}$ & $\begin{array}{l}\text { End of thermal input from } \\
\text { quantum gravity due to prior } \\
\text { universe quantum bounce. } \\
\text { Brane theory predicts massive } \\
\text { relic graviton production }\end{array}$ & $\begin{array}{l}\text { Axion wall is in process of } \\
\text { disappearing due to mark rise in } \\
\text { temperatures. Quintessence } \\
\text { valid for short time interval }\end{array}$ & $\begin{array}{l}\text { Wheeler formula for relic } \\
\text { graviton production produces } \\
\text { massive spike gravitons due to } \\
\text { sharp rise in temperatures }\end{array}$ \\
\hline \multirow[t]{2}{*}{ Time $0<t \approx t_{P}$} & $\begin{array}{l}\text { Relic graviton production } \\
\text { largely tapering off, due to } \\
\text { thermal input rising above a } \\
\text { preferred level, via brane } \\
\text { theory calculations. Beginning } \\
\text { of regime where the } \Lambda_{4-\text { Dim }}\end{array}$ & $\begin{array}{l}\text { Axion wall disappears, and } \\
\text { beginning of Guth style } \\
\text { inflation. Quintessence scalar } \\
\text { equations are valid . Beginning } \\
\text { of regime for } \\
\frac{\Lambda_{4-\text { dim }}}{\Delta}-1 \approx \frac{1}{n}\end{array}$ & \multirow[t]{2}{*}{$\begin{array}{l}\text { Wheeler formula for relic } \\
\text { graviton production leading to } \\
\text { few relic gravitons being } \\
\text { produced. }\end{array}$} \\
\hline & $\begin{array}{l}\text { is associated with Guth style } \\
\text { inflation. }\end{array}$ & $\begin{array}{l}\left|\Lambda_{5-\operatorname{dim}}\right| \\
5 \mathrm{dim}\end{array}$ & \\
\hline
\end{tabular}

Also, one can expect a difference in the upper limit of Park's four dimensional inflation value for high temperatures, onn the order of 10 to the 32 Kelvin, and the upper bound, as Barvinsky (2006) predicts. If put into the Harkle-Hawking's wave function, this diffenence is equivalent to a nucleation-quantization condition, which, it is claimed, is a way to delineate a solution to the cosmic landscape problem that Guth $(1981,2000,2003) 38 \cdot 39 \cdot 40$ discussed. In order to reference this argument, it is useful to note that Barvinsky in ( 2006) came up with

$$
\left.\Lambda_{\max }\right|_{\text {Barvinsky }} \cong 360 \cdot m_{P}^{2}
$$

A minimum value of

$$
\left.\Lambda_{\text {min }}\right|_{\text {Barvinsky }} \cong 8.99 \cdot m_{P}^{2}
$$

This is in contrast to the nearly infinite value of the Planck's constant as given by Park (2003)

$$
\cdot \Lambda_{4-\mathrm{dim}} \text { is defined by Park (2003).with } \varepsilon^{*}=\frac{U_{T}^{4}}{k^{*}} \text { and } U_{T} \propto(\text { external temperature }) \text {, and } k^{*}=\left(\frac{1}{\text { 'AdScurvature }}\right) \text { so }
$$
that

$$
\left.\Lambda_{4-\operatorname{dim}, \text { Max }}\right|_{\text {Park }} \underset{T \mapsto 10^{32} \text { Kelvin }}{\longrightarrow} \infty
$$

As opposed to a minimum value as given by Park (2003)

$$
\cdot \Lambda_{4-\mathrm{dim}}=8 \cdot M_{5}^{3} \cdot k^{*} \cdot \varepsilon^{*} \underset{\text { external temperature } \rightarrow 3 \text { Kelvin }}{\longrightarrow}(.0004 \mathrm{eV})^{4}
$$

Table 2. What can be said about cosmological $\Lambda$ in 5 and 4 dimensions

\begin{tabular}{cccc}
\hline $\begin{array}{c}\text { Time } \\
0 \leq t<<t_{P}\end{array}$ & Time $0 \leq t<t_{P}$ & Time $0<t \approx t_{P}$ & Time $t>t_{P} \rightarrow$ today \\
\hline$\left|\Lambda_{5}\right|$ undefined, $T \approx \varepsilon^{+} \rightarrow$ & $\left|\Lambda_{5}\right| \approx \varepsilon^{+}$, & $\left|\Lambda_{5}\right| \approx \Lambda_{4-\text { dim }}$ & $\left|\Lambda_{5}\right| \approx$ huge, \\
$T \approx 10^{32} K$ & $\Lambda_{4-\text { dim }} \approx$ extremely large & $T$ smaller than & $\Lambda_{4-\text { dim }} \approx$ small, \\
$\Lambda_{4-\text { dim }} \approx$ almost $\infty$ & $T \approx 10^{12} K$ & $T \approx 10^{12} K$ & $T \approx 3.2 K$ \\
\hline
\end{tabular}




\section{This leads to presenting the Wheeler graviton production formula for relic gravitons}

As is well known, a good statement about the number of gravitons per unit volume with frequencies between $\omega$ and $\omega+d \omega$ may be given by (assuming here, that $\bar{k}=1.38 \times 10^{-16} \mathrm{erg} /{ }^{0} \mathrm{~K}$, and ${ }^{0} \mathrm{~K}$ is denoting Kelvin temperatures, wherewhere Gravitons have two independent polarization states), as given by Weinberg (1972).

$$
n(\omega) d \omega=\frac{\omega^{2} d \omega}{\pi^{2}} \cdot\left[\exp \left(\frac{2 \cdot \pi \cdot \hbar \cdot \omega}{\bar{k} T}\right)-1\right]^{-1}
$$

Thee hypothesis presented here is that input thermal energy (given by the prior universe) inputted into an initial cavity/region (dominated by an initially configured low temperature axion domain wall) would be thermally excited to reach the regime of temperature excitation. This would permit an order-of-magnitude drop of axion density $\rho_{a}$ from an initial temperature $\left.T_{d S}\right|_{t \leq t_{P}} \sim H_{0} \approx 10^{-33} \mathrm{eV}$.

\section{Graviton power burst/ where did the missing contributions to the cosmological 'constant' parameter go?}

To do this, one needs to refer to a power spectrum value that can be associated with the emission of a graviton. Fortunately, the literature contains a working expression of power generation for a graviton produced for a rod spinning at a frequency per second $\omega$, per Fontana 41 (2005), for a rod of length $\widehat{L}$ and of mass $m$ a formula for graviton production power. This is a variant of a formula given by Park 42(1955), with mass $m_{\text {graviton }} \propto 10^{-60} \mathrm{~kg}$

$$
P(\text { power })=2 \cdot \frac{m_{\text {graviton }}{ }^{2} \cdot \widehat{L}^{4} \cdot \omega_{\text {net }}{ }^{6}}{45 \cdot\left(c^{5} \cdot G\right)}
$$

The contribution of frequency here needs to be understood as a mechanical analogue to the brute mechanics of graviton production. The frequency $\omega_{\text {net }}$ is set as an input from an energy value, with graviton production number (in terms of energy) derived via an integration of Eq. (7) above, $\widehat{L} \propto l_{P}$. This value assumes a huge number of relic gravitons are being produced, due to the temperature variation.

$$
\langle n(\omega)\rangle=\frac{1}{\omega(\text { net value })} \int_{\omega 1}^{\omega 2} \frac{\omega^{2} d \omega}{\pi^{2}} \cdot\left[\exp \left(\frac{2 \cdot \pi \cdot \hbar \cdot \omega}{\bar{k} T}\right)-1\right]^{-1}
$$

And then one can set a normalized "energy input "as $E_{\text {eff }} \equiv\langle n(\omega)\rangle \cdot \omega \equiv \omega_{\text {eff }}$; with $\hbar \omega \longrightarrow{ }_{\hbar \equiv 1}^{\longrightarrow} \omega \equiv\left|E_{\text {critical }}\right|$, which leads to the following table of results, where $T^{*}$ is an initial temperature of the pre- inflationary universe condition 43 .

Table 3. Graviton burst

\begin{tabular}{cc}
\hline Numerical values of graviton production & Scaled Power values \\
$\mathrm{N} 1=1.794 \times 10^{-6}$ for Temp $=T^{*}$ & Power $=0$ \\
$\mathrm{~N} 2=1.133 \times 10^{-4}$ for Temp $=2 T^{*}$ & Power $=0$ \\
$\mathrm{~N} 3=7.872 \times 10^{+21}$ for $T e m p=3 T^{*}$ & Power $=1.058 \times 10^{+16}$ \\
$\mathrm{~N} 4=3.612 \times 10^{+16}$ for $T e m p=4 T^{*}$ & Power $\cong$ very small value \\
$\mathrm{N} 5=4.205 \times 10^{-3}$ for $T e m p=5 T^{*}$ & Power $=0$ \\
\hline
\end{tabular}

Here, N1 refers to a net graviton numerical production value as given by Eqn. (9). There.T is a distinct power spike of thermal energy that is congruent with a relic graviton burst.

41 Fontana, G., "Gravitational Wave Propulsion," in CP746, Space Technology and Applications International Forum-STAIF 2005, editede by M. S. El-Genk, American Institute of Physics, Melville, NY, 2005

42 Park, D, "Radiations from a Spinning Rod,”Physical Review, 99, No. 4, 1324-1325 (1955).

43 Beckwith, A.W., "Does A Randall-Sundrum Brane World Effective Potential Influence Axion Walls Helping to Form a Cosmological Constant Affecting Inflation?" http://www.citebase.org/cgi-bin/citations?id=oai:arXiv.org:gr-qc/0603021 (2006), in AIP Conf.Proc.880:1170-1180 (2007). 


\section{Appendix VIII: Using our bound to the cosmological constant to link relic graviton production to branes}

We use our bound to the cosmological constant to obtain a conditional escape of gravitons from an early universe brane. To begin, we present using the paper written by Leach et al on conditions for gravitaton production 44

$$
B^{2}(R)=\frac{f_{k}(R)}{R^{2}}
$$

Also there exists an 'impact parameter'

$$
b^{2}=\frac{E^{2}}{P^{2}}
$$

This leads to, practically, a condition of 'accessibility' via $R$ so defined is with respect to 'bulk dimensions'

$$
\begin{gathered}
b \geq B(R) \\
f_{k}(R)=k+\frac{R^{2}}{l^{2}}-\frac{\mu}{R^{2}}
\end{gathered}
$$

Here, $\mathrm{k}=0$ for flat space, $\mathrm{k}=-1$ for hyperbolic three space, and $\mathrm{k}=1$ for a three sphere, while an radius of curvature

$$
l \equiv \sqrt{\frac{-6}{\Lambda_{5-\operatorname{dim}}}}
$$

Here, we have that we are given

$$
k^{*}=\left(\frac{1}{\text { 'AdS curvature }}\right)
$$

Park et al note that if we have a 'horizon' temperature term

$$
U_{T} \propto(\text { external temperature) }
$$

We can define a quantity

$$
\varepsilon^{*}=\frac{U_{T}^{4}}{k^{*}}
$$

Then there exists a relationship between a four-dimensional version of the $\Lambda_{\text {eff }}$, which may be defined by noting

$$
\Lambda_{5-\mathrm{dim}} \equiv-3 \cdot \Lambda_{4-\mathrm{dim}} \cdot\left(\frac{U_{T}}{k^{* 3}}\right)^{-1} \propto-3 \cdot \Lambda_{4-\mathrm{dim}} \cdot\left(\frac{\text { external temperature }}{k^{* 3}}\right)^{-1}
$$

So

$$
\Lambda_{5-\operatorname{dim}} \underset{\text { external temperature } \rightarrow \text { small }}{\longrightarrow} \text { very large value(10) }
$$

In working with these values, one should pay attention to how $\cdot \Lambda_{4-\operatorname{dim}}$ is defined by Park, et al.

$$
\cdot \Lambda_{4-\operatorname{dim}}=8 \cdot M_{5}^{3} \cdot k^{*} \cdot \varepsilon^{*} \underset{\text { external temperature } \rightarrow 3 \text { Kelvin }}{\longrightarrow}(.0004 \mathrm{eV})^{4}
$$

Here, I am defining $\Lambda_{5-\text { dim }}$ as being an input from changes in the actual potential system due to 


$$
\Lambda_{5-\text { dim }} \equiv-3 \cdot \Lambda_{4-\operatorname{dim}}(\Delta V) \cdot\left(\frac{U_{T}}{k^{* 3}}\right)^{-1}
$$

Here we are looking at how the initial vacuum energy 'cosmological constant' parameter may be effected by a change in the potential system with the $\Lambda_{4-\operatorname{dim}}(\Delta V)$ tern with different temperature values implied for input into the four dimensional vacuum energy. I.e. $\Lambda_{4-\text { dim }}(\Delta V)$ starts off with a given temperature value input as we look at $(\Delta V)$ for a maximized potential value, and subsequently dropping as the potential system evolves to a different value as inflation proceeds.

This, for potential, $(\Delta V)$ is defined via transition between the first and the second potentials of the form given by

$$
V \equiv V_{0}-c_{3} \phi^{3}+\frac{1}{2} \lambda \cdot \phi^{2} \cdot \rho^{2}+\ldots
$$
Sarkar treated the inflaton as having a varying effective mass, with an initial value of effective mass of $m_{\phi}^{2}=\frac{d^{2} V}{d \phi^{2}}$ given
a before and after phase transition value of

$$
m_{\phi}^{2}=-\left.6 c_{3} \cdot\langle\phi\rangle\right|_{\text {Before-phase-transition }} \underset{\text { phase-transition }}{\longrightarrow}-6 c_{3} \cdot\langle\phi\rangle+\left.\lambda \cdot \Sigma^{2}\right|_{\text {after-phase-transition }}
$$

Either this potential can be used, or we just use a variant of a transition to the Race track potential given by

$$
\begin{aligned}
& V(s, \phi)=\frac{1}{2 s} \cdot\left(A \cdot\left(2 s+N_{1}\right) \cdot e^{-s / N_{1}}+B \cdot\left(2 s+N_{2}\right) \cdot e^{-s / N_{2}}\right)^{2} \\
& +\frac{1}{s} \cdot A \cdot B \cdot\left(2 s+N_{1}\right) \cdot\left(2 s+N_{2}\right) \cdot e^{-s / N_{1}} \cdot e^{-s / N_{2}} \cdot(1-\cos (\phi \cdot \varepsilon))
\end{aligned}
$$

This with a version of the scalar field in part be minimized. This is assuming that we are having $s \rightarrow N_{a} \neq \infty$, leading to minima for $\phi_{k}=k \pi / \varepsilon$, with $\mathrm{k}$ being the positive and negative integers, i.e. this helps delineate between two condensates. If we have a complex scalar field $\phi_{j}=X_{j}+i \cdot Y_{j}$. we have moduli arguments which add far more structure . Either type of structure can be used and put in so we come up with an effective value for a potential system. I.e. at a given

$$
B_{e f f}^{2}\left(R_{t}\right)=\frac{1}{l_{\text {eff }}^{2}}+\frac{1}{4 \cdot \mu}
$$

Claim : $R_{b}(t)=a(t)$ ceases to be definable for times $t \leq t_{P}$ where the upper bound to the time limit is in terms of Planck time and in fact the entire idea of a de Sitter metric is not definable in such a physical regime.

\section{REFERENCES}

[1] Padmanabhan, T, “ Theoretical Astro physics, Volume 1 : Astrophysical processes “, Cambridge University Press, Cambridge, UK, 2000

[2] Mathur, S., and Chowdhury, B., “Fractional Brane States in the Early Universe”, Classical and Quantum Gravity, 24 (2007), pp. $2689-2720$

[3] Wesson, P., “ Space-Time-Matter, Modern Kaluza Klein Theory”, World Scientific, Singapore, Republic of Singapore, 1999

[4] Lifschytz, G. “ Black Hole thermalization rate from brane anti brane model “, arXIV hep-th//0406203 v1 23 june, 2004

[5] Smoot, G, 11th Paris Cosmology Colloquium, August 18th, 2007 with respect to Smoot, G, “ CMB Observations and the Standard Model of the Universe" 'D.Chalonge' school, http://chalonge.obspm.fr/Programme2007.html

[6] Kolb, E., and Turner, S. “ The Early Universe”, Westview Press, Chicago, USA, 1994

[7] Weinberg, S., Gravitation and Cosmology: Principles and Applications of the General theory of Relativity, John Wiley \& Sons, Inc. New York, 1972

[8] Lloyd, S., "Computational capacity of the universe”, Phys. Rev. Lett. 88, 237901 (2002)

[9] Beckwith, A. "Symmetries in evolving spacetime from prior to present unverses", arXIV math-ph/0501028 (2007)

[10] Park, D.K., Kim, H., and Tamarayan, S., "Nonvanishing Cosmological Constant of Flat Universe in Brane world Senarios,” Phys.Lett. 
B535 (2002) pp. 5-10

[11] Barvinsky,A., Kamenschick,A., Yu, A. , “ Thermodynamics from Nothing: Limiting the Cosmological Constant Landscape, Phy Rev D 74121502 (Rapid communications)

[12] Padmanabhan, T., “An Invitation to Astro physics”, World Scientific Publishing Co. Pte. Ltd, Singapore, 2006

[13] Padmanabhan, T.” Understanding Our Universe: Current Status and Open Issues”, in 100 Years of Relativity Space-Time Structure: Einstein and Beyond, edited by Ashtekar, A., World Press Scientific, Singapore, 2005

[14] Crowell, L, “Quantum Fluctuations of Space Time “, World Scientific Series in Contemporary Chemical Physics, Vol 25, World Scientific, PTE, LTD, 2005, Singapore

[15] Misner, C., Thorne, K., and Wheeler, J.,” Gravitation”, W.H. Freeman, and Company, San Francisco, USA, 1973

[16] Wesson, P., “ Space-Time-Matter, Modern Kaluza Klein Theory”, World Scientific , Singapore, Republic of Singapore, 1999

[17] Mukhanov, V., "Physical foundations of Cosmology", Cambridge University Press, Cambridge, UK, 2005

[18] Frampton, P, Baum, L, “Turnaround in Cyclic Cosmology “,Phys.Rev.Lett. 98 (2007) 071301 , arXiv:hep-th/0610213v2

[19] Dowker,H. F.,"Causal sets and the deep structure of spacetime”, arXIV gr-qc/0508109v1 26 Aug 2005; in 100 Years of Relativity Space-Time Structure: Einstein and Beyond, edited by Ashtekar, A., World Press Scientific, Singapore, 2005

[20] Ruutu, V. , Eltsov, V, Gill, A., Kibble, T., Krusius, M., Makhlin, Y.G., Placais, B., Volvik, G, and Wen, Z., "Vortex Formation in neutron - irradiated 3He as an analog of cosmological defect formation," Nature 382, 334-336 (25 July 1996)

[21] Lalak, Z., Ross, G., and Sakar, S., "Racetrack Inflation and assisted Moduli Stabilization", arXIV hep-th / 0503178 v 2, 12 June, 2006

[22] http://www-thphys.physics.ox.ac.uk/user/SubirSarkar/

[23] Blanco-Pillado, J., Burgess, C., Cline, J., Escoda, C., Gomez - Reino , M., Kallosh, R., Line, A., Quevedo, F., “Racetrack Inflation”, JHEP 0411 (2004) 076, arXIV hep-th / 0411011

[24] Carroll, S., Spacetime and Geometry:: An Introduction to General Relativity, Addison Wesley Co., San Francisco, 2005

[25] Private communications with Subir Sarkar received as of January 2998

[26] Durrer, R., "Cosmological Pertubation theory", From the book "Physics of the Early Universe", in Lecture Notes in Physics, 653", with Papantonopoulos, E., as editor and printed by Springer- Verlag 2004

[27] Hunt, P. and Sakar, S., “ Multiple Inflation and the WMAP “glitches” “, Phys Rev D Volume 70, 103518

[28] Sanchez, N. 11th Paris Cosmology Colloquium, August 18th, 2007 with respect to Sanchez, N., "Understanding Inflation and Dark Energy in the Standard Model of the Universe", 'D.Chalonge' school

[29] Ruutu, V. , Eltsov, V, Gill, A., Kibble, T., Krusius, M., Makhlin, Y.G., Placais, B., Volvik, G, and Wen, Z., "Vortex Formation in neutron - irradiated 3He as an analog of cosmological defect formation," Nature 382, 334-336 (25 July 1996)

[30] Enqvist, K., Mazumdar, A., Perez--Lorenzana, A., “ Dumping inflaton energy out of this world”,Phys Rev D, Volume 70, 103508

[31] H. V. Peiris, H., Komatsu, E., Verde, L., Spergel, D., Bennett, C., Halpern, M., Hinshaw, G., Jarosik, N., Kogut, A., Limon, M., Meyer, S., Page, L., Tucker, G., Wollack, E., and Wright, E.," First-Year Wilkinson Microwave Anisotropy Probe (WMAP) Observations: Implications For Inflation", The Astrophysical Journal Supplement Series, 148:213-231, 2003 September

[32] Lecture notes taken at IGC conference, http://www.gravity.psu.edu/igc/conf_files/program_complete.pdf with respect to the Penrose presentation, "Conformal Cyclic cosmology, Dark Matter, and Black Hole Evaporation", plus questions asked of the lecturer in the aftermath of that presentation

[33] Brax, Ph., Davis, A., Davis, S., Jeannerot, R., Postman, M., “ D-term Uplifted Racetrack Inflation”, Journal of Cosmology and Astroparticle Physics, JCAP01(2008) 08

[34] Samtleben, D., Staggs, S., Winstein, B., "The Cosmic Microwave Background for Pedestrians: A Review for Particle and Nuclear Physicists”, Annu..Rev . Nucl. Part. Sci. 2007, 57, : 245-83

[35] Zhitinisky, A., "Dark Matter as Dense Color Superconductor' arXIV: astro-ph/0204218 v1 12 April 2002. Refer to the last version of this document

[36] Rothman, T. and Boughn, S., “Can Gravitons be Detected ?”, Foundations of Physics, 36, No. 12,, 1801-1825 (December 2006), arXIV gr-qc/0601043 v 202 Jan 2006

[37] Beckwith, A.W, "How can Brane World physics influences the formation of a cosmological constant relevant to graviton production?" arXIV physics/0612010, accessible as http://arxiv.org/abs/physics/0612010, E.J T.P., 4, No. 15, 105-142 (July 2007a). 
[38] Guth, A.H "Inflationary Universe: A Possible Solution to the Horizon and Flatness Problems," TTPhys. Rev. TTD 23, $347-356$ (1981)

[39] Guth. A.H., "Inflation and Eternal Inflation,” http://www.citebase.org/cgi-bin/citations?id=oai:arXiv.org:astro-ph/0002156 (2000).

[40] Guth. A.H., “Eternal Inflation,” http://online.itp.ucsb.edu/online/strings_c03/guth/pdf/KITPGuth_2.pdf (2003

[41] Fontana, G., "Gravitational Wave Propulsion," in CP746, Space Technology and Applications International Forum-STAIF 2005, editede by M. S. El-Genk, American Institute of Physics, Melville, NY, 2005

[42] Park, D, “Radiations from a Spinning Rod,”Physical Review, 99, No. 4, 1324-1325 (1955).

[43] Beckwith, A.W., "Does A Randall-Sundrum Brane World Effective Potential Influence Axion Walls Helping to Form a Cosmological Constant Affecting Inflation?” http://www.citebase.org/cgi-bin/citations?id=oai:arXiv.org:gr-qc/0603021 (2006), in AIP Conf.Proc.880:1170-1180 (2007).

[44] Leach, J.A. and Lesame, W.M ," Conditional escape of gravitons from the brane” arXIV gr-qc/0502109 v326 Apr 2005 